\title{
Advances and Perspectives in the Use of Carbon Nanotubes in Vaccine Development
}

\author{
Elidamar Nunes de Carvalho \\ Lima $^{1,2}$ \\ Ricardo Sobhie Diaz $\mathbb{D}^{2}$ \\ João Francisco Justo ${ }^{3}$ \\ José Roberto Castilho Piqueira' \\ 'Telecommunication and Control \\ Engineering Department, Polytechnic \\ School of the University of São Paulo, São \\ Paulo, Brazil; ${ }^{2}$ Infectious Diseases \\ Division, Department of Medicine, \\ Federal University of São Paulo, São \\ Paulo, Brazil; ${ }^{3}$ Electronic Systems \\ Engineering Department, Polytechnic \\ School of the University of São Paulo, São \\ Paulo, Brazil
}

\begin{abstract}
Advances in nanobiotechnology have allowed the utilization of nanotechnology through nanovaccines. Nanovaccines are powerful tools for enhancing the immunogenicity of a specific antigen and exhibit advantages over other adjuvant approaches, with features such as expanded stability, prolonged release, decreased immunotoxicity, and immunogenic selectivity. We introduce recent advances in carbon nanotubes (CNTs) to induce either a carrier effect as a nanoplatform or an immunostimulatory effect. Several studies of CNTbased nanovaccines revealed that due to the ability of CNTs to carry immunogenic molecules, they can act as nonclassical vaccines, a quality not possessed by vaccines with traditional formulations. Therefore, adapting and modifying the physicochemical properties of CNTs for use in vaccines may additionally enhance their efficacy in inducing a $\mathrm{T}$ cellbased immune response. Accordingly, the purpose of this study is to renew and awaken interest in and knowledge of the safe use of CNTs as adjuvants and carriers in vaccines.
\end{abstract}

Keywords: carbon nanotubes-CNTs, functionalization, nanoplatform, nanovaccine, immunomodulation, carrier effect

\section{Introduction}

\section{Nanotechnology and Nanovaccines}

Nanotechnology refers to the production of components on the order of magnitude of 1-100 $\mathrm{nm}$ in diameter. The concept that nanoparticles (NPs) can be used in human health being a subject of great interest, both for medical diagnosis and imaging and for immunotherapy, especially regarding nanovaccines. ${ }^{1}$ Nanovaccines are used to induce an immune response to infectious agents and consist of nanoscale formulations based on particles whose components generate a desired immune response. ${ }^{2}$

Nanotechnology makes it possible to design and manufacture nanostructures and introduce them into the human body, improving health and repairing cellular structures at the molecular level. This ability is very useful in biological applications and can be used to facilitate immunotherapy. ${ }^{3}$ Therefore, it is a promising possibility aiding in health restoration. ${ }^{1}$ This capability is due to the unique capacity of nanomaterial properties that interact with cellular functions in different ways. ${ }^{1,3}$

Vaccines represent one of the most significant medical advances of modern civilization in centuries, allowing unparalleled public health achievements. ${ }^{4}$ For infectious diseases, almost all vaccines are prophylactically administered, which is fundamental for the control of several diseases, inducing an appropriate and robust immune response. ${ }^{5}$ Vaccine development is historically based on Louis Pasteur's principles of "isolate, inactivate and inject". Initially, vaccines were produced with 
attenuated or inactivated microorganisms such as viruses and bacteria. The main feature of therapeutic vaccines is their elimination of the effects caused by pathogens. ${ }^{6}$

Modern vaccines use subunits derived from the pathogen in question; however, both vaccines (classical and modern) need a specific delivery system to activate their effects on the immune system. ${ }^{4}$ Nanotechnology has a unique role in designing vaccines called nanovaccines, promoting specific and powerful immune responses. ${ }^{7}$ A common characteristic of nanovaccines, in contrast to conventional vaccines, is the possibility of including antibodies and/or other immune molecules that can generate a specific and robust immune response. $^{8}$

Nanovaccines have unique characteristics, regarding their efficiency in modulating the immune response. This efficiency is especially important for small adjuvant molecules that are quickly eliminated from the bloodstream. ${ }^{9}$ NPs measuring from 10 to $100 \mathrm{~nm}$ enter the extracellular matrix and travel to the lymphatic ganglia, where they can be internalized by resident macrophages and activate $\mathrm{T}$ cell responses that are fundamental to promoting a robust immune response. Adjuvant and antigen delivery using NPs is considered useful to extend exposure in lymphoid organs such as the lymph nodes, producing prolonged and vigorous immune responses. $^{3,10}$

Consequently, nanovaccines are more efficient than conventional vaccines because in addition to inducing humoral and cellular immune responses, this response is more robust and specific. ${ }^{2}$ Other essential characteristics of nanovaccines are i) a longer duration of antigen presentation by dendritic cells (DCs), ii) an increased ability to elicit cellular and humoral immunity, iii) an increased ability to stimulate cytokine production, iv) the ability to protect adjuvants and antigens from early degradation by proteolytic enzymes, v) the potential to include different molecules that can potentiate the immune response, vi) the ability for low-dose administration, and vii) the absence of a requirement for refrigeration (Figure 1). ${ }^{2}$ All of these features are essential and must be considered when designing nanovaccines; however, the main advantage of nanovaccine is the ability to include different immune molecules, adjuvants and antigens and to potentiate and modulate/orchestrate the desired immune response. ${ }^{11-13}$

\section{Achievements in Nanotechnology Applied to Vaccines}

Despite the achievements of traditional vaccines in preventing various diseases, HIV infections, coronavirus

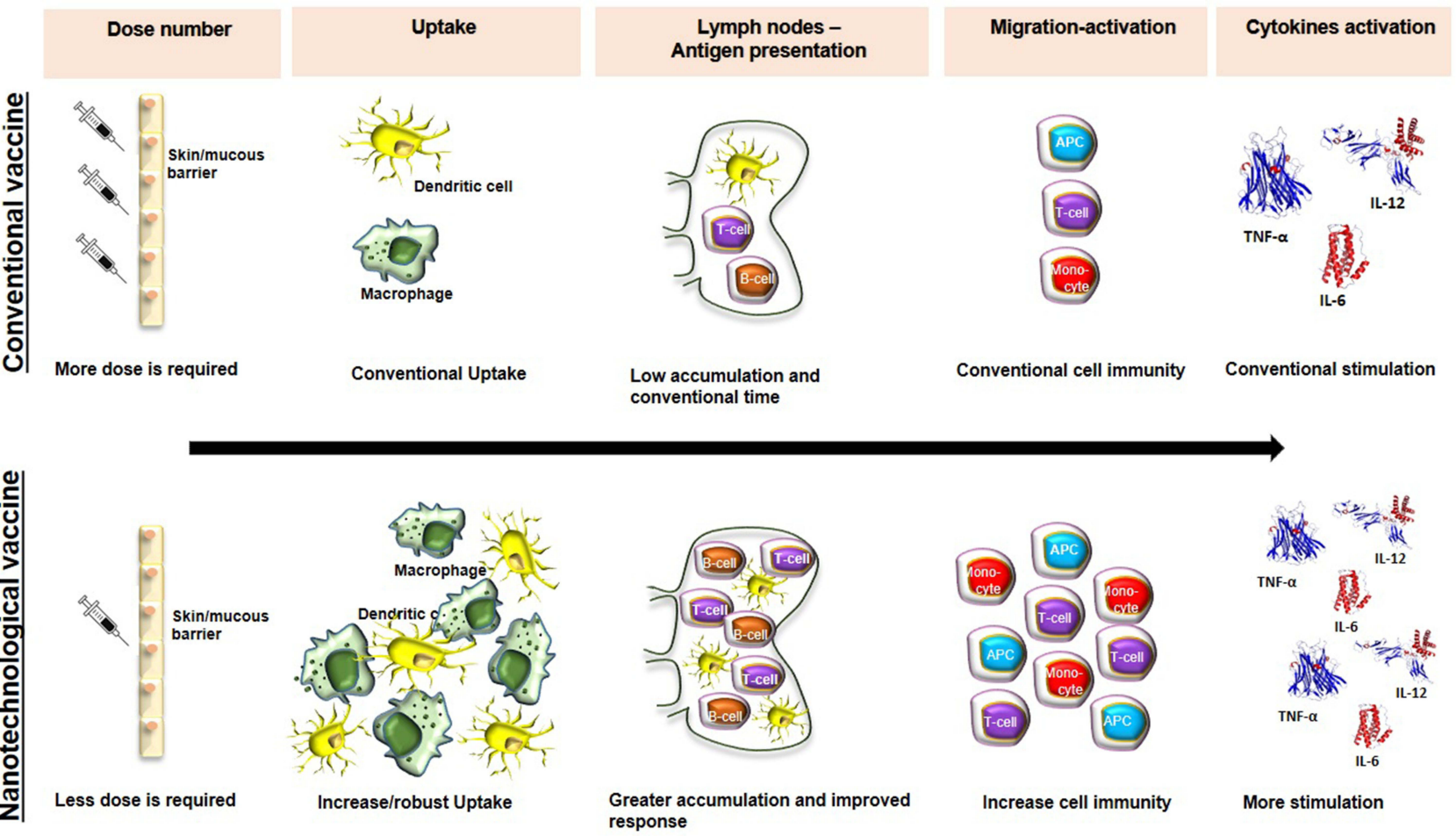

Figure I Comparative immune activation between conventional vaccines and nanovaccine. Nanovaccines, compared to conventional vaccines, are efficient in inducing a more robust humoral and cellular immune response. 
disease 2019 (COVID-19) and influenza, new immunotherapeutic/vaccine approaches are needed. ${ }^{14}$ In this regards, nanovaccines have the characteristics of multifunctionality, adaptability, increased antigenic stability, targeted delivery and long-term release of antigens, culminating in more efficient and robust immune responses, in addition to being advantageous in minimizing aspects related to inflammation and toxicity due to adjuvants. ${ }^{13}$

Flexibility in the design of nanovaccines reduces many of the main challenges encountered in the development of conventional vaccines and constitutes a fundamental advance in vaccine/immunotherapeutic applications. Nanovaccines being developed in a multifaceted manner, allowing the presentation of different classes of antigens, which contributes to increased immunostimulatory action. ${ }^{11,13}$ The possibility of coupling multiple antigens, a strategy called a nanoplatform, appears to be one of the greatest achievements of nanotechnology applied to vaccines in the treatment of various infections/diseases. ${ }^{10}$

One of the significant achievements of nanotechnology is the application of antitumor nanovaccines, which present robustness and immunological efficiency, surpassing the applications of conventional immunotherapy., ${ }^{3,8}$ Nanovaccines can be structured in their design to allow the encapsulation of adjuvants and antigens, potentially stimulating the immune system by increasing the CD8+ T-cell response, which is essential in the defense against cancer. $^{15,16}$

A variety of cancer-fighting nanoplatforms are being investigated/developed for molecular delivery into lymphoid organs, tissues and cells, with the aim of promoting potent, long-lasting, antitumor immunity and reducing the adverse effects of conventional immunotherapy. ${ }^{17,18}$ Thus, one of the advances is antitumor therapy using nanovaccines based on ovalbumin (OVA) as a modulating antigen and a STAT3 siRNA to activate DCs through Toll-like receptor 7 (TLR7). The results obtained show that these multivalent antigen-carrying nanovaccines and adjuvants induce elevated cytokine levels and tumor volume reduction. ${ }^{19}$ Previous studies using a cancer nanovaccine based on the neoantigen peptide (Adpgk) associated with TLR 7/8 (agonist R848) and TLR9 (agonist CpG) showed increased immunogenicity, reduced acute toxicity, and regression of tumor volume by $70 \%{ }^{15}$

Another advancement obtained by nanotechnology in nanovaccines is the use of liposomes as immunostimulatory molecules. ${ }^{20}$ Coadministration of cationic liposomes associated with trehalose 6,6-dibehenate (TDB) and the influenza vaccine induces increased cellular and humoral immune responses, promoting a robust $\mathrm{T}$ helper 1 (Th1) response and elevated levels of IL-1 $\beta$, IL- 2 , IL-12, IFN- $\gamma$ and TNF- $\alpha .{ }^{21}$ Nanovaccines based on liposomes can induce the formation of germinal centers and promote the synthesis of specific antibodies with long-term efficacy. Accordingly, Moon et al demonstrated that a nanovaccine using malaria antigen induced protective immunity against Plasmodium vivax sporozoites, with appreciably higher titers and more balanced Th1/Th2 responses, compared with vaccines based on soluble protein mixed with monophosphoryl lipid A (MPLA). This nanovaccine was based on MPLA, which is associated with the malaria antigen VMP001 and incorporated into liposomes. $^{22}$

\section{Advances in the Defense Against Influenza, HIV and Coronavirus Infections}

Nanotechnology provides technological approaches with results and perspectives in the treatment of several pathologies, including viral infections such as influenza, human immunodeficiency virus (HIV) and coronavirus. ${ }^{23-26}$ Influenza infection threatens global health and constitutes a burden to public health systems due to its different viral strains/genomic variability. ${ }^{27}$ Accordingly, influenza vaccines are currently produced to cover the different viral strains circulating. $^{28}$

One approach used in the treatment of influenza is the use of viral hemagglutinin (HA)-associated protein. Previous studies have shown that a nanovaccine based on viral HA inserted at the interface of ferritin subunits inhibited hemagglutination with increased antibody titers compared to those achievable with the traditional vaccines in use and triggered neutralization of antibodies specific for two vulnerable and conserved HAs. ${ }^{29}$ This strategy of self-assembling proteins not only showed improved immune potency against the influenza virus but also advanced insight into the design of nanovaccines for different viral infections. The use of layered protein NPs associated with HA and M2 antigens induces a longlasting, robust immune response, according to results obtained by Lei Deng research group. ${ }^{30}$

The resulting immune response of this nanovaccine is the induction of long-lasting antibodies and protection against challenges caused by influenza viruses. A breakthrough in influenza nanovaccines is Vaxfectin $^{\circledR}{ }^{31}$ Its cationic, liposome-encapsulated 
formulation with activity against herpesvirus type 2 (HSV2 ) is also used for immunization against the H5N1 influenza virus. Another achievement in nanovaccines for influenza is Inflexal $^{\circledR}, 32$ which is an FDA-approved formulation based on influenza virus HA surface molecules fused with lipid components. ${ }^{24,33}$

Nanotechnology has also been a great aid against HIV. The HIV-1 pandemic has claimed more than 30 million people since the beginning of the infection. By 2020, approximately 36.2 million people were living with HIV1 , with 1.7 million newly infected. ${ }^{34}$ Highly active antiretroviral therapy (HAART), despite being a strategy for the treatment of acquired immunodeficiency syndrome (AIDS) caused by HIV, has barriers in regard to HAART resistance. Although many antiretroviral drugs have actions in treating HIV infection, none can eliminate the viral reservoirs. ${ }^{35}$ Therefore, the need to develop a vaccine to contain the HIV-1 pandemic has become critical; however, even after more than three decades of research, there is no effective vaccine. Several factors affect the development of a vaccine capable of preventing infection with HIV-1, but the most salient aspect is that the viral genome is extremely dynamic, variable, and highly mutable due to the action of the reverse transcriptase. ${ }^{36}$

Nanotechnology has achieved unprecedented advances throughout the history of HIV, allowing the design of functional structures to treat this infection. Several approaches have been developed to obtain an efficient vaccine against HIV, including nanovaccines based on nanocapsules, ${ }^{8}$ Nanocrystals, ${ }^{37}$ lipid NPs, nanocarriers, liposomes and micelles. ${ }^{17,22,38}$ The use of biomimetic nanovaccines, can be designed with properties that directly modulate the immune system to defend against viral infections. These nanovaccines exhibit high encapsulation efficiency or coupling of antiretroviral drugs, cytokines, and enzymes as well as drug delivery to desired/specific sites. $^{39}$

Previous studies have shown that the use of liposomes facilitates endocytosis into cells of the mononuclear phagocytic system (MPS), allowing the contents to reach the HIV-infected reservoir. ${ }^{40}$ The results obtained by Hanson et al demonstrated that an HIV nanovaccine based on the membrane proximal region (MPER)-associated MPLA and the stimulator of interferon genes (STING) agonist cyclic-di-GMP (cdGMP) induced a significant humoral immune response in addition to an increased T-cell response. $^{41}$ Another study used the HIV viral surface glycoprotein gp120/gp41 as a component of VLP nanovaccines against HIV. The vaccine showed profound modulation of the immune system and germinal center maintenance from hypermutation of B cells, resulting in the secretion of highly neutralizing anti-HIV antibodies. ${ }^{42}$

An efficient strategy to combat HIV infections is to coat NPs with the viral cell membrane. Previous results have shown increased production of neutralizing antibodies against HIV infection. ${ }^{43}$ Additionally, after repeated antigen presentation, multivalent nanovaccines/nanoplatforms against HIV infection tend to increase the retention of vaccine particles before they reach the germinal center, which enhances B-cell activation. ${ }^{39,44}$ Work developed by Wahome et al made the association in the outer MPER of HIV-1 gp41, aiming to produce a helical conformation of the 4 E10 $\alpha$ epitope. The results showed increased titers of antibodies specific to membrane regions, indicating that this conjugate exhibits potential as a nanovaccine against HIV. $^{45}$

Nanotechnology has also been used to seek solutions for infections with the coronavirus that causes severe acute respiratory syndrome (SARS). ${ }^{23,46}$ The pandemic of COVID-19 (caused by SARS-CoV-2) has spread exponentially worldwide since November 2019, when the first case was diagnosed in Wuhan, China. ${ }^{47}$ Since then, the development of immunotherapies that can contain the rapid spread of this infection has become critical and urgent. Several studies are being carried out to develop new vaccines against COVID-19; however, aspects beyond prevention, such as efficient treatment and diagnosis, are also challenging. ${ }^{48}$

Advances in nanotechnology have led to the development of new strategies for the treatment and prevention of COVID-19, including nanovaccines to control infection. Several approaches, including attenuated viruses, particulate subunits, nucleic acids, and recombinant viral vectors, have been used in the development of nanovaccines. Most studies use structural proteins such as the spike (S), envelope (E) or membrane (M) proteins to act as inducers of neutralizing antibodies. ${ }^{46,49}$

Nanovaccines are increasingly being used as new strategies and potential therapeutic approaches, aiming at the prevention, treatment, and diagnosis of different COVID19 infections. $^{23}$ A widely used approach is the use of nanoformulations/NPs, whose unique characteristics, for example, include potential for drug encapsulation that surpasses that of conventional therapeutic options. The use of a polyglycolic acid (PLGA) capsid as a cationic STING associated with a recombinant MERS-CoV antigen 
adjuvant to promote Th1 immune and cellular responses against MERS-CoV infection increases antigen-specific cellular and humoral concentrations. ${ }^{50}$

Nanoformulations allow the controlled and sustained release of antigens/therapeutic agents, which interfere with viral entry into cells, improving the success of preventive and therapeutic measures against COVID-19. Nanoformulations composed of $\mathrm{S}$ surface proteins and M1 matrix proteins have been shown to induce robust and effective immune responses with the production of neutralizing antibodies against MERS-CoV blocking its replication in the lungs of vaccinated mice. ${ }^{51}$

Another approach exploiting nanotechnology to address COVID-19 is the use of polymeric micelles. ${ }^{52}$ Single-chain polymers conjugated to specific ligands, which help to engulf or coat the virus, result in neutralization and destabilization of the viral genome. This approach/strategy used coronavirus surface ligands to develop a nanotechnology/nanovaccine called a NanoViricide ${ }^{\circledR}$. 53

Raghuwanshi et al encapsulated the plasmid DNA encoding protein $\mathrm{N}$ (pVAXN) of SARS-CoV in biotinylated chitosan NPs for nasal immunization. The biotinylated chitosan NPs were surface functionalized with a recombinant bifunctional fusion protein (bfFp) containing the singlestranded variable fragment ( $\mathrm{scFv}$ ) for DEC-205 receptors. The positively charged chitosan NPs efficiently bound to negative sialic residues in the mucosa of the nasal region. ${ }^{54}$

Most studies using nanotechnology approaches to combat COVID-19 are in the early or developmental stages, and problems will remain until these vaccine systems can progress to clinical use. The efficacy, stability, and safety of nanoscale-based systems for both prevention and diagnosis must be evaluated to ensure their clinical relevance. ${ }^{55}$ Several studies are in progress to allow approaches using nanotechnology to offer interesting systems that can address major public health challenges, promoting achievements not only in the prevention but also in the diagnosis and treatment of different infections, especially influenza, HIV and COVID-19. ${ }^{23,25,39}$

\section{How Do Carbon Nanotubes Interact with Cellular Structures? Properties of Carbon Nanotubes}

Carbon nanotubes (CNTs) are cylindrical structures that are formed by carbon atoms. CNTs are classified according to the number of walls that compose them as singlewalled CNTs (SWCNTs) or multiwalled CNTs
(MWCNTs). They can be generated by electrical discharge and laser ablation using graphite target vaporization or synthesized through the deposition of vapor containing catalytic metal). ${ }^{56}$

They have an approximate diameter of $0.4-2 \mathrm{~nm}$ (SWCNTs) or 10-100 nm (MWCNTs). Both types of CNTs can be used in biological biomedical systems, provided that they are treated to minimize and/or eliminate toxic effects, making them biocompatible. ${ }^{57}$ Reducing the size and diameter of CNTs, a fundamental characteristic for biological applications, can be achieved by acid and ultrasound treatment and purification methods. ${ }^{58}$

The CNT structure allows the coupling of different molecules, including antigens, DNA and drugs, which can subsequently be targeted for the therapeutic treatment of numerous pathologies or for the detection of diseasecausing pathogens. ${ }^{59}$ In this way, due to their magnetic, electronic, mechanical and optical properties, CNTs are to a promising group of nanomaterials. ${ }^{60}$

\section{Uptake and Interaction with Cellular Structures}

The entry of CNTs into cells occurs mainly through internalization mechanisms, such as direct translocation by NP insertion or diffusion by endocytic-phagocytic mechanisms. ${ }^{61}$ The mechanisms by which CNTs are taken up into cells include phagocytosis, diffusion and endocytosis. CNT phagocytosis is a process that occurs by active digestion within small vesicles named phagosomes, which are temperature-dependent, a process that requires energy expenditure via ATP. ${ }^{62}$ These types of entry are related to factors such as CNT synthesis, size and type of functionalization, as well as other physicochemical characteristics of the nanomaterials. ${ }^{63-65}$ Although CNT entry mechanisms depend on their size, diameter and functionalization groups, size is the predominant factor. CNTs Between 100-200 nm can enter the cell via clathrin-coated internalization mechanisms, and CNTs smaller than $100 \mathrm{~nm}$ are internalized into vesicles via caveolae (Figure 2) ${ }^{63}$

Macrophages play a critical role in the immune response and constitute the organism's first line of defense against foreign particles. Studies have shown that CNT entry into macrophages results in cell death, suggesting that this death occurs via rupture of the plasma membrane. ${ }^{66}$ The direct interaction of CNTs with the plasma membrane is partially mediated by the association of macrophage receptors with 


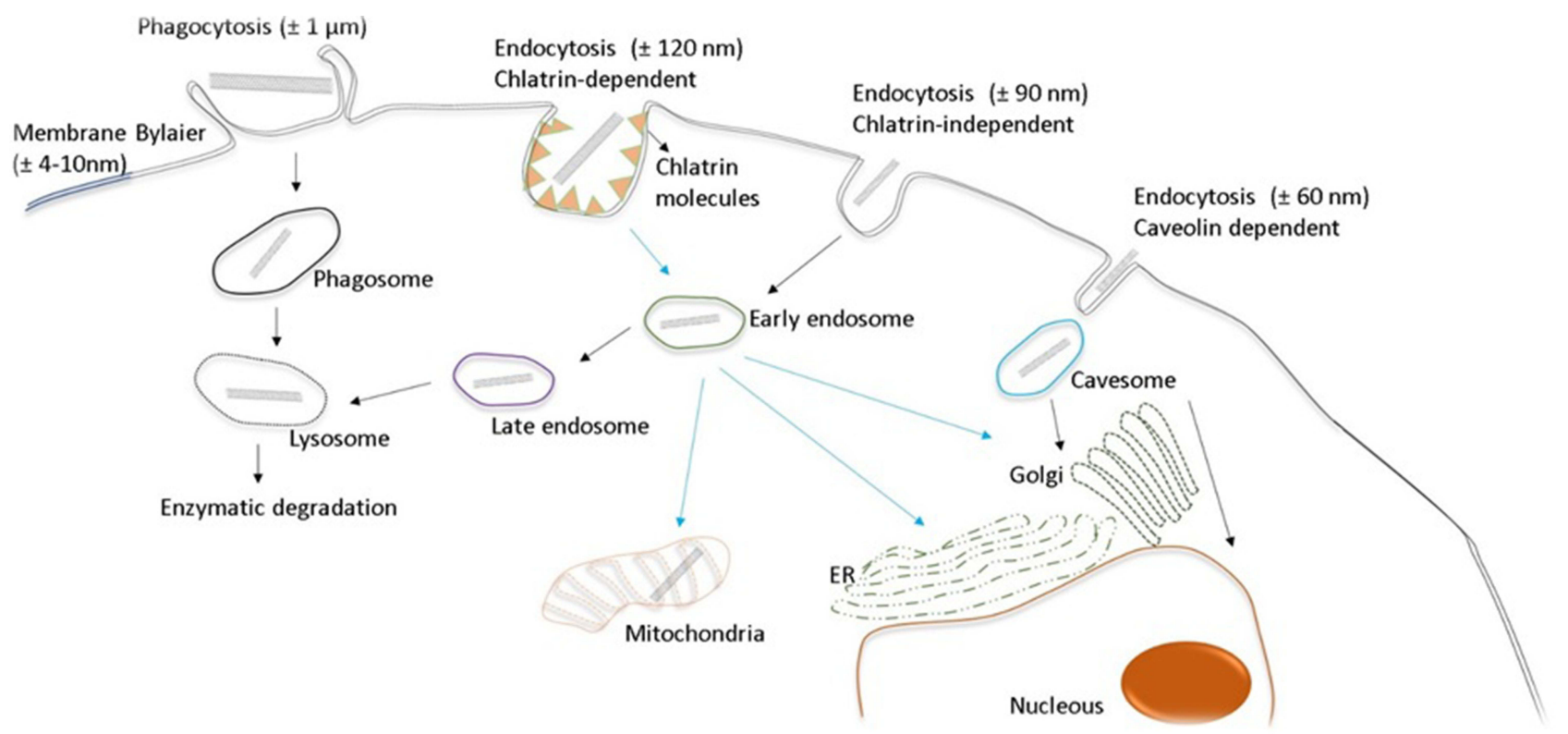

Figure 2 CNT uptake and interaction with the cell structures. The size of the nanotubes is the main factor for their entry into the cell. CNTs between I00-200 nm can enter the cell by clathrin-coated internalization mechanisms, and CNTs smaller than $100 \mathrm{~nm}$ are internalized into vesicles. Phagocytosis of CNTs occurs by active digestion within small vesicles called phagosomes.

collagenous structures (MARCOs) on the cell surface, suggesting that CNTs bind to macrophages via MARCOs, which stimulates cell membrane extension, causing membrane disruption and eventually cell death. ${ }^{67}$

CNTs are engulfed by phagocytic cells through several phagocytic and endocytic mechanisms. Previous studies have shown that CNT uptake by macrophages occurs when they are exposed to a CNT concentration of $10 \mu \mathrm{g} /$ $\mathrm{mL}$ for 2-4 days. Thus, CNTs are found in macrophage lysosomes two days after uptake, where the entry of CNTs into cells is not efficient when the size of these nanomaterials is greater than $10 \mu \mathrm{m} .{ }^{68} \mathrm{CNTs}$ can affect membrane polarity in macrophage mitochondria, suggesting that these signaling events can induce transcription factors such as NF- $\kappa \beta$, culminating in the activation of inflammatory processes and cytokine production. ${ }^{64}$

CNT internalization is also cell lineage dependent. In this sense, CNT internalization by epithelial cells is related to the size of these NPs, and particles larger than $10 \mu \mathrm{m}$ undergo frustrated internalization. ${ }^{65}$ Active and passive mechanisms of CNT cellular uptake culminate in accumulation within organelles and/or in elimination. CNTs cross the plasma membrane very efficiently by endocytic or passive diffusion, penetrating the cell membrane and accumulating in different organelles/cellular structures. ${ }^{69}$

CNTs can be internalized by the endocytic pathway inside vesicles called endosomes before being directed to lysosomes in the perinuclear compartment. The mechanisms of CNT cellular uptake are energy-dependent and occur in a clathrin-dependent manner for both SWCNTs and MWCNTs (Figure 2). ${ }^{63}$ However, SWCNTs temporarily remain inside lysosomes in $\mathrm{pH}$-dependent environments. ${ }^{70} \mathrm{CNTs}$ penetrate cells by passive diffusion through nanoneedle penetration mechanisms caused by diffusion of single CNTs through the plasma membrane. ${ }^{71}$ According to computational studies, CNTs penetrate the lipid bilayer of the plasma membrane via the following steps: i) landing and fluctuation of CNTs on the cell surface; ii) penetration of the CNTs across the heads of lipidic groups in the cytoplasmic membrane; and iii) sliding of the CNTs through the lipidic components of the cytoplasmic membrane. ${ }^{72}$ Another factor to be considered in cellular uptake mechanisms is that endocytic mechanisms are involved in internalizing CNT clusters, while isolated CNTs are internalized by membrane diffusion. ${ }^{71}$

Functionalized CNTs (CNT-f) with phospholipidpolyethylene glycol (PL-PEG), cross the lipid membrane of nonphagocytic cells by passive diffusion and accumulate in the mitochondria and in macrophage lysosomes. Phagocytosis mechanisms are dependent on the physicochemical properties of nanotubes and the phagocytic nature of the cells. Yet another factor to be considered in cellular uptake mechanisms is the CNT size. ${ }^{65}$ Studies have shown that CNTs larger than $400 \mathrm{~nm}$ are found in 
endocytic vesicles and that CNTs smaller than $400 \mathrm{~nm}$ are scattered throughout the cytoplasm. ${ }^{63,73}$

CNTs can be visualized inside cells by optical, electron and fluorescence microscopy. Optical microscopy is ideal for analyzing cells in vivo, which allows the identification of internalized nanomaterials. Electron microscopy improves the spatial resolution of CNT images; however, the cells need to be fixed, unlike fluorescence microscopy, which detects CNTs indirectly by detecting molecules coupled to them. ${ }^{74}$ When used as a drug delivery system (DDS), CNTs represent an essential molecular transport vehicle for epitopes, including vaccination systems. ${ }^{17}$

The exceptional capacity of CNTs to enter cells drives their use as application systems for both drugs and vaccines. However, it is necessary to analyze and quantify their effects in different organs and cellular structures to ensure the safe use of these nanomaterials. ${ }^{75,76}$ The interaction and uptake of CNTs at the cell surface depend on the type of functionalization present on the CNT surface and the phagocytic nature of the cell (Figure 3). ${ }^{77}$ Therefore, the treatment/functionalization of CNT surfaces is crucial for interactions with cellular membranes, since the ambiguity of these nanomaterials can induce cytotoxicity or activation of specific molecules. ${ }^{77,78}$

Entry mechanisms and intracellular elimination are the objects of intensive research aimed at modeling and adapting these properties of nanomaterials to enhance their biocompatibility. CNTs accumulate around the cell nucleus in HeLa and $3 \mathrm{~T} 3$ cells, where they are dispersed with DNA. ${ }^{79}$ CNT-f

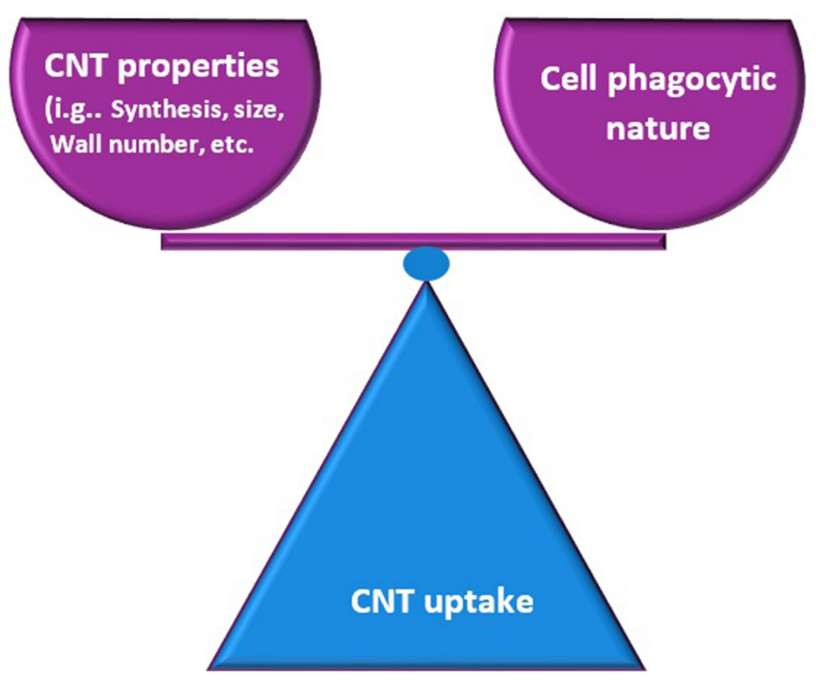

Figure 3 CNT uptake is mediated according their characteristics. The entry and interaction of the CNT is dependent on the balance between different characteristics such as the phagocytic nature of the cells, size and types of functionalization of the nanotubes. with PL-PEG, crosses the plasma membrane of nonphagocytic cells, such as COS7 and MCF7 EVC304 cells, by passive diffusion, later accumulating in mitochondria. ${ }^{73,80}$

In phagocytic cells such as macrophages and RAW264 cells, CNTs accumulate in lysosomes. ${ }^{81}$ These studies show that the mechanisms of CNT internalization by phagocytosis depend not only on the physicochemical properties of these nanomaterials but also on the phagocytic nature of the cells (Figure 3). ${ }^{77}$ These characteristics can be modified or engineered via functionalization of CNTs and alteration of some characteristics that can be modulated, especially in relation to biocompatibility, through many types of functionalization on the CNT surface. In this context, biocompatibility can be enhanced when CNTs are treated with acids (1,3-dipolar, oxidation and amidation). ${ }^{82}$

\section{Activation of Signaling Pathways Induced by Carbon Nanotubes}

Most studies of the cellular internalization of CNTs under in vitro conditions report the use of progenitor/myeloid cells, including macrophages, eosinophils and DCs, which recognize foreign matter through membrane receptors such as TLRs and, through this binding of receptors and foreign matter, activate signaling pathways that culminate in the immune response. ${ }^{58}$

The immune activation pathways induced by CNTs are related to several characteristics, including the physicochemical properties of these nanomaterials, which can be modified through functionalization processes, and any modifications lead to different immune reactions. ${ }^{16}$ In this regard, cell signaling pathways are altered/orchestrated by the physicochemical properties of CNTs, which include the different possibilities of modifications/functionalizations on the surfaces of these nanomaterials, potentially directing biological activities. ${ }^{72}$ Physicochemical surface modifications of CNTs (eg, the addition of carboxyl groups or amines) culminate in diverse immune responses. In this context, depending on the surface modification of the nanotubes, it is possible not only to alter the cellular uptake of these nanomaterials but also to alter the binding receptors involved and, consequently, to activate different cellular signaling pathways. ${ }^{83}$

The cellular effects of CNT uptake are related to their recognition of plasma membrane receptors and activation of immune cells. Nanotubes are easily internalized by cells by energy-dependent and independent mechanisms, and after cellular uptake of this nanomaterial, they target 
different structures and organelles, interacting with cytoplasmic proteins. This hydrophobic nature of the nanotube surface can induce the binding of protein domains rich in hydrophobic amino acids, and carboxylated nanotubes regulate $\mathrm{BMP}^{84}$

Mario Pescatori research group investigated the immune activation pathways after the uptake of different types of CNTs (ox-MWCNT-1, ox-MWCNT-NH3+-1, and ox-MWCNT-NH3+-2). ${ }^{85}$ The nanotubes tested activated monocyte-related immune pathways and are therefore referred to as monocyte-activating nanotubes (MA-CNTs) . These nanotubes induced activation of IL6 and CD40, DC maturation, tumor necrosis factor (TNF)-a/TNFR1-2 and NFKB signaling, and Th1 chemokine production (via CXCR3 and CCR5 ligands). Nanotubes (MA-CNTs) are strongly associated with immune transcripts, which include genes encoding CCR5 and CXCR3 ligands. These signaling pathways, especially Th1, are activated during acute inflammatory processes and pathogen clearance, which are critical in controlling tumor rejection. These results show that the use of CNTs as immunotherapeutic molecules is promising in cancer treatment. ${ }^{58}$

The functionalization of CNTs is a key factor in the modulation/orchestration of specific cytokines, eg, anionic functionalization (carboxylated and PEGylated CNTs), decreases the production of cytokines and growth factors IL-1beta and activates TLR, IL-6, TNF, NFKB, DC maturation, chemokines via Th1 and CXCR3 and CCR5 ligands. ${ }^{85}$ TLR receptors on the surface of phagocytic cells detect CNTs and, once they are phagocytosed by macrophages, induce chemokine secretion through TLR2/ 4-MyD88-NF- $\kappa \mathrm{B}$ signaling. TLRs activate adaptor protein signaling pathways, including MyD88 differentiation factors, which induce transcription factors such as NF-kB and subsequent cytokine/chemokine production. ${ }^{86}$

However, phagocytosis of nonfunctionalized CNTs, which are cytotoxic, activates IL-1 beta and caspase-1 and various inflammatory cytokines through pathways associated with oxidative stress and the caspase cascade. $^{87}$ CNTs functionalized with ammonium groups by 1.3-dipolar cycloaddition, when phagocytosed by human monocytes, induce the expression of CD25 and markers of IL1 beta, IL6, TNF, and IL10 without activating cytotoxic mechanisms. Therefore, functionalization/ modification of CNTs with ammonium groups or oxidation promotes profound modulation of immune-regulatory pathways without activating apoptotic pathways. ${ }^{88}$
CNT activation culminates in a profound modulation of inflammatory molecules at the transcriptomic level, including TLR, IL-6, DC maturation, TNF, NFKB and the Th1 chemokine pathway (CXCR3 and CCR5). ${ }^{85}$ Due to their potential to direct the synthesis of immunological molecules and their critical role in inflammatory pathways, depending on the functionalization, CNTs are presented as molecules of excellence in the immunotherapy scenario and emerge as a potential adjuvant against several pathologies, including infectious diseases caused by HIV, influenza and COVID. ${ }^{25,39,75}$

\section{Biodegradation and Elimination of Carbon Nanotubes}

Enzymatic degradation of CNTs occurs under abiotic conditions. Allen et al in 2008 was first to described nanotube biodegradation by horseradish peroxidase (HRP), an enzyme of vegetal origin that causes the oxidation of these nanomaterials by enzymatic oxidation, and as a result, CNT length is reduced. ${ }^{89}$

CNT-f with PEG has the capacity for neutrophil activation, inducing an increase in myeloperoxidase (MPO) and hypochlorous acid production, which favors their autodegradation. The same effects occur in the presence of MPO, which is of animal origin and expressed in neutrophils, and eosinophil peroxidase (EPO). ${ }^{90}$

The biodegradation of CNTs occurs due to the action of enzymes such as lignin peroxidases, xanthine oxidases and manganese peroxidases, which demonstrates the degradative capacity of these nanomaterials. Oxidized CNTs can be degraded by the plant enzyme HRP over a period of ten days. Daily degradation also occurs in the presence of hydrogen, which shortens CNTs and induces the production of polyaromatic oxides and, finally, $\mathrm{CO}_{2}{ }^{91,92}$ Previous studies have shown that in vivo, macrophages pretreated with enzyme inhibitor targeting both MPO and reactive oxygen species (ROS) exhibit increased degradation processes. ${ }^{68}$

In this regard, the absence of NADPH enzymes decreases CNT degradation, showing a clear dependency on the biodegradation of these nanomaterials by macrophages. NADPH oxidase-dependent ROS generation plays a crucial role in CNT biodegradation by macrophages. ${ }^{89,93}$ CNTs can be degraded by macrophages, and this degradation mechanism is related to the increase in oxygen when CNTs are internalized by the cell, raising the consumption of NADPH. ${ }^{68}$ CNT biodegradation by macrophages 


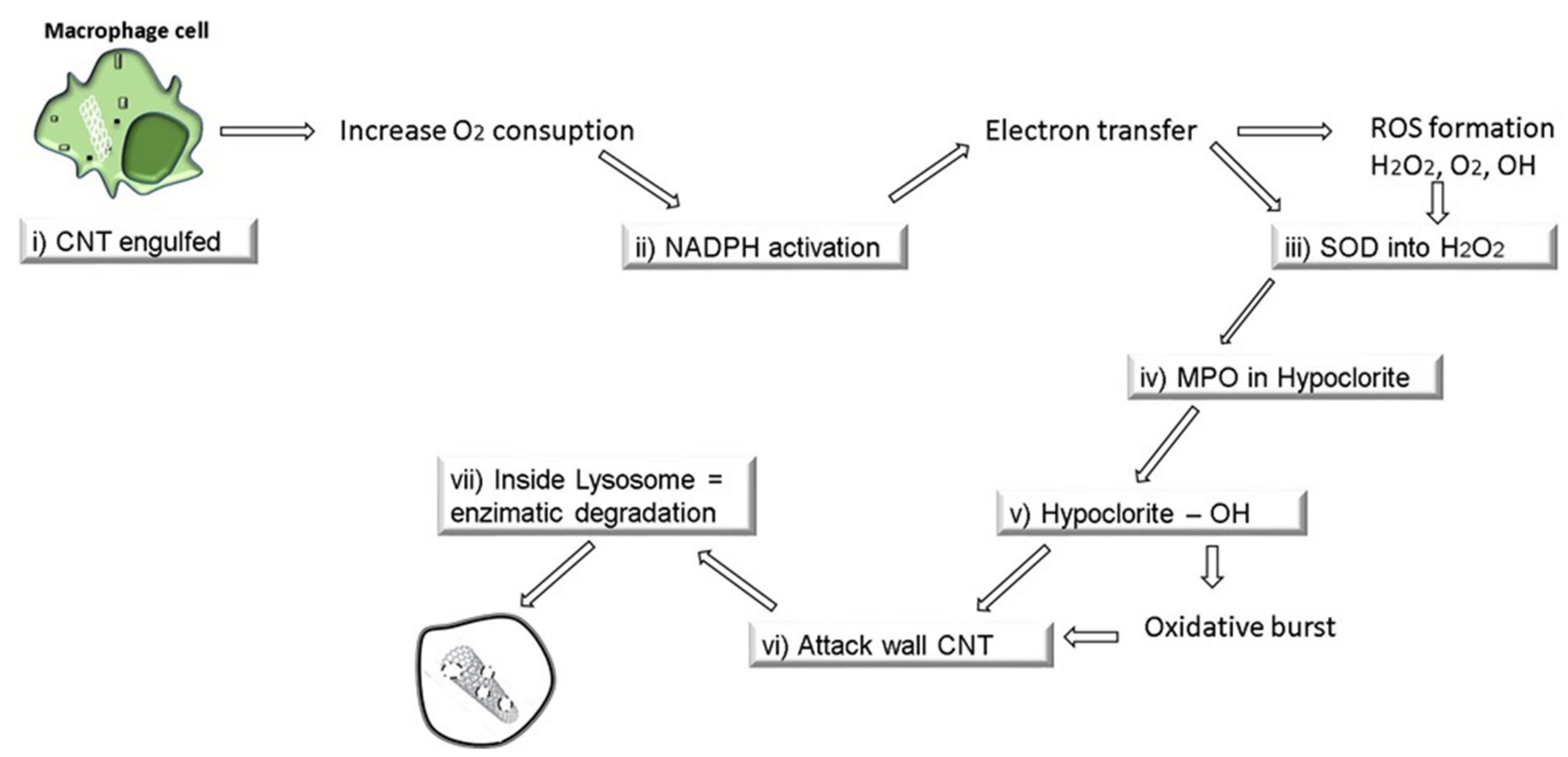

Figure 4 CNT biodegradation by macrophages cell and elimination. The biodegradation mechanisms are depending on enzymatic processes with the participation of particle degrading oxidases in an event called oxidative burst.

follows the steps below: i) CNTs are internalized by macrophages; ii) NADPH oxidase is activated to generate $\mathrm{O}_{2}$; iii) superoxide dismutase (SOD) converts superoxide into $\mathrm{H}_{2} \mathrm{O}_{2}$ or free radicals such as nitric oxide (NO) to generate ONOO-; and iv) MPO combines $\mathrm{H}_{2} \mathrm{O}_{2}$ with $\mathrm{Cl}$ to produce hypochlorites or, in the presence of $\mathrm{Fe} 3+, \mathrm{H}_{2} \mathrm{O}_{2}$ is converted to $\mathrm{OH}$, and hypochlorites and $\mathrm{OH}$ can attack the CNT walls, creating holes in the structure and resulting in degradation by carbon dioxide, a process called oxidative burst (Figure 4). ${ }^{95}$ Thus, all biodegradation mechanisms are dependent on enzymatic processes. ${ }^{93-95}$

After biodegradation, CNTs can be eliminated by exocytosis, an essential mechanism in CNT elimination. Endosomes in the trans-Golgi complex are responsible for the extrusion of these nanomaterials, but it is an elimination process that occurs under cell stress conditions. ${ }^{63}$ Several factors influence the degradation rate of CNTs, including the size of the nanomaterial and wall thickness; for example, SWCNTs are degraded faster than MWCNTs. The CNT diameter becomes a decisive factor in the degradation time and efficiency of these nanomaterials, and the functionalization of the CNTs, ie, the chemical treatment of their surface, is a significant factor that directly influences the degradation rate of CNTs. ${ }^{96}$ Biodegradation is related to several factors, including the type of functionalization, the size of the CNTs, the varieties of molecules attached to these nanomaterials, the experimental conditions used, and especially the type of cells and immune recognition. In summary, the rate of biodegradation and elimination of CNTs is dependent on a combination of all the elements mentioned above. ${ }^{94}$

\section{Nanotubes Mechanisms to Activate Immune System \\ Cellular Immune Response-Activation of Macrophages and Monocytes}

The immune system is composed of organs, tissues and cells that maintain health. The balance of these components governs homeostasis in response to different infections, controlling the balance between health and disease in an organism. ${ }^{97}$ It is essential to analyze the features related to the safety and development of new materials used to restore and modulate these immunological systems in patients affected by diseases, such as by changing specific pathways involved in the immune response. ${ }^{98}$ The possibility of applying research on nanotechnology within the criteria for inducing immunity with nanomaterials supports the development and performance of these tools, the objective of which is to modulate the immune response that can be orchestrated according to the nanomaterial's surface treatment. ${ }^{2,19,84}$ In this context, Nanotechnology allows nanomaterials to develop within the criteria of safe innovation, allowing their safe application. $^{75}$ It is possible to create products based on the physical-chemical properties of nanomaterials. 
The first line of cellular defense in the human body comprises phagocytes, cells capable of internalizing pathogens or other foreign elements. Monocytes, macrophages and neutrophils are among the first cells to recognize CNTs, which later enter the cytoplasmic vesicles. Monocytes play an important role in eliminating and destroying pathogens, acting in both innate and adaptive immunity and presenting immunological properties in the production of various model molecules, such as cytokine responses. ${ }^{86,87}$

The introduction of nanomaterials whose physicalchemical parameters can be used in the orchestration of the immune system has made possible the application of CNTs with immunomodulation potential. Immunological effects are directly facilitated by the possibility of modeling the design of different properties of CNTs, including size and functionalization, that will serve as a basis for the desired biological/biomedical application. In this context, understanding nanomaterial interactions with the immune system facilitates the development of specific strategies for immunotherapies. ${ }^{19,55}$

\section{Activation of Dendritic Cells}

DCs specialize in presenting antigens circulating in the bloodstream. Previous studies have shown that CNTs produced by high-pressure CO (HiPCO nanotubes) induce lung inflammation, facilitating the recruitment of DCs to lung tissues with poor DC activation. ${ }^{99}$ However, with CNT-f, this process culminates in a $\mathrm{T}$ cell-suppressive response. ${ }^{88,100}$ Different types of CNT functionalization can alter DC recognition and profiles and, consequently, antigen presentation and processing for T cells. ${ }^{60} \mathrm{CNT}-\mathrm{f}$ with anionic phospholipids drastically changed recognition and uptake by a wide range of phagocytic cells, including DCs. Additionally, studies performed by Villa et al demonstrated that CNT-f coupled with tumor protein induces a specific IgG immune response together with this peptide, showing that CNTs can be used as antigens with antigenpresenting cells (APCs). ${ }^{98}$

Another important characteristic is that both the kinetic mechanism of nanotube uptake and intracellular nanotube trafficking by DCs involve immediate uptake, with a median time of five minutes. This rapid and robust absorption is consistent with mechanisms involved in macropinocytosis, which is a fundamental mechanism of macromolecular antigen uptake in DCs. Additionally, according to previous results, DCs can internalize a large number of nanotubes without compromising cell integrity. ${ }^{99,100}$

\section{Lymphocyte Activation}

Previous studies have shown that the effects of CNTs on lymphocytes are related to the morpho-physical-chemical characteristics of nanomaterials and the type of functionalization. ${ }^{101}$ Although CNTs do not affect cytokine secretion, they increase the dose-dependent secretion of TNF- $\alpha /$ IL-12/IL-6 and IL-2/IFN- $\gamma$. CNT-f with a 1.3-dipolar cycloaddition reaction are not toxic to murine $\mathrm{T}$ and $\mathrm{B}$ cells and may stimulate $\mathrm{T}$ cell lines more efficiently when associated with specific molecules such as CD3 ${ }^{102}$ Furthermore, CNT-f with amphotericin $\mathrm{B}$ can better induce an antifungal effect than amphotericin $\mathrm{B}$ alone in $\mathrm{T}$ cell lines. ${ }^{103}$

The global impact of CNTs on immune responses was investigated after injection into mice and led to the conclusion that CNT administration improves immune responses. In this manner, the activation of cells of the innate immune system, such as monocytes/macrophages, is the first event, resulting in the secretion of proinflammatory cytokines, which stimulates Th cells. ${ }^{104}$ All these events are important for the differentiation of B cells into antibody-secreting cells (Figure 5). ${ }^{100}$ This sequence of events has been shown using nonfunctionalized CNTs, called pristine CNTs (PCNTs), injected intraperitoneally or intravenously with OVA as the antigen. OVA-specific antibody production was increased, suggesting a critical immunostimulatory effect of CNTs. ${ }^{105}$

This sequence of events (inflammation leading to lymphocyte activation) may be related to the allergic response observed when PCNTs are intratracheally instilled in mice. Many studies have analyzed the activation of immune cells by CNTs, but few studies have focused on the immunogenicity of CNTs themselves. ${ }^{106}$ Notably, the induction of anti-CNT antibodies has not been shown to date, even after injection into mice in the presence of a robust immunological adjuvant. ${ }^{107}$ This immune neutrality is particularly desirable when CNTs or any other NPs are to be used as carriers for drug administration. Thus, various physicalchemical parameters, such as size, surface load, hydrophobicity, surface topography and material composition, ${ }^{108}$ can be amplified and optimized to facilitate activation interactions to orchestrate the immune response (Figure 6). ${ }^{108}$

\section{Activation of the Complement System and Interleukins}

Another component of the immune response is the complement system, composed of over 40 proteins in the 


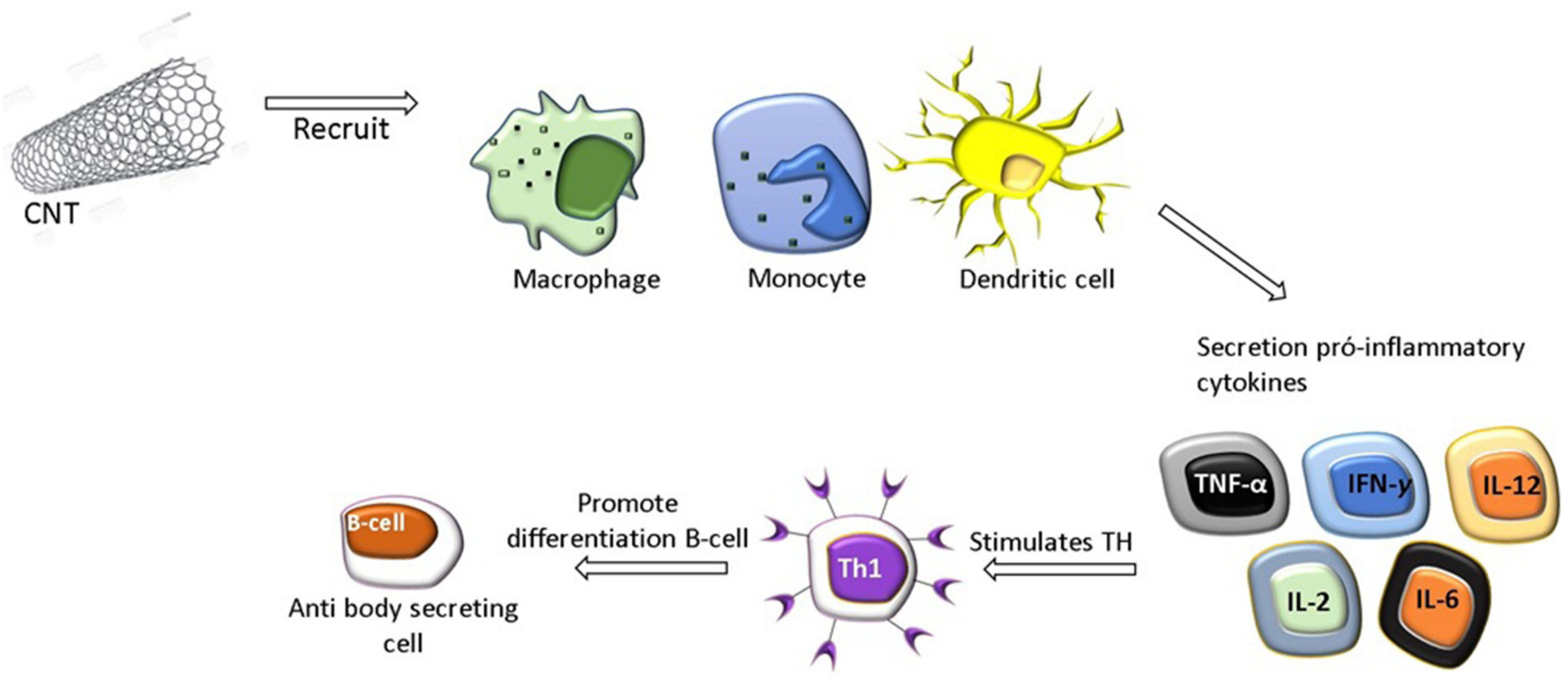

Figure 5 CNT Immunological properties and activation of immune cells. CNT activate the first line of defense of the innate immune system, including cells such as monocytes/macrophages, resulting in the activation of pro-inflammatory cytokines, which stimulates Th cells. All of these events are important for the differentiation of B cells into secretory cells of antibodies.

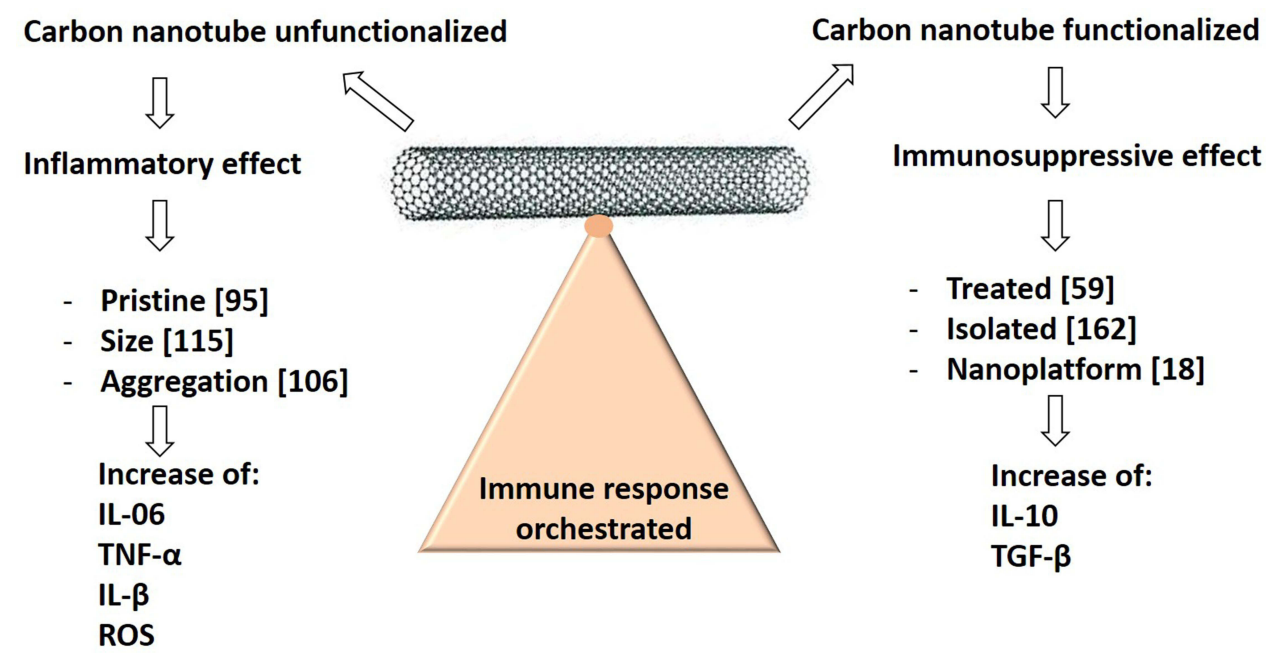

Figure 6 CNT Immunological activation or suppression based on functionalization. Different physicochemical parameters influence the toxicity and immune stimulatory aspects of CNTs, including hydrophobicity, size, surface charge, and CNT composition that can be modified and optimized to facilitate the orchestration of the immune response.

Notes: Modified from Fadel TR, Fahmy TM. Immunotherapy applications of carbon nanotubes: from design to safe applications. Trends Biotechnol. 20I4;32(4): I98-209, Copyright 2014 , with permission from Elsevier. ${ }^{75}$

cytoplasm and on the cell surface. The complement system allows the recognition of harmful microorganisms as well as changes such as apoptosis, necrosis and aggregate proteins, among others. Therefore, when NPs are administered, they are immediately covered by a crown composed of proteins, among which the complement system recognizes abnormal components, including pathogens and synthetic materials. ${ }^{109}$

Activation of the complement system can be mediated by the induction of three pathways, the classic, alternative and lectin pathways, which converge around the formation of $\mathrm{C} 3$ convertase. This protease activates $\mathrm{C} 3$, cleaving it into $\mathrm{C} 3 \mathrm{~b}$. The degradation of $\mathrm{C} 3 \mathrm{~b}$ and its byproduct, $\mathrm{iC} 3 \mathrm{~b}$, is the key to mediating the link between the target and phagocytic cells. Some complement proteins, such as C1q, are sensors that recognize foreign components and, depending on the recognition of these invading components, can activate the three distinct pathways. ${ }^{110}$ All three pathways are part of the complement cascade system, whose objective is to eliminate the pathogen/target by 
forming the membrane attack complex (MAC) or by mediating indirect opsonization, and the activation of the complement system by both the classical and alternative pathways was reported using PCNTs. CNT-f with PEG, an enzyme that provides stability to the nanomaterial in the blood circulation, can attenuate complement system recognition. ${ }^{111}$

The activation of these pathways of the complement system depends on the type of CNT functionalization (eg, PEGylated CNT-f activates the lectin pathway, and CNT-f with albumin activates the classical and alternative pathways via $\mathrm{C} 1-\mathrm{q}) .{ }^{112}$ In this context, $\mathrm{C} 1 \mathrm{q}$ proteins from the classical pathway bind CNTs without additional activation. The potential of CNTs to induce an immune activation response to the complement system must be considered. ${ }^{113}$ CNTs can be modulated by modification/functionalization of their surface for further use in medicine/biology. ${ }^{85,114}$

CNTs can stimulate the secretion of proinflammatory cytokines such as IL-1 $\beta$, TNF- $\alpha$, IL- 8 and IL-6, inducing a robust inflammatory response. PCNTs activates both the classical and alternative pathways of the complement system. However, when CNTs are functionalized with PEG, the alternative pathway with cleavage of $\mathrm{C} 4$ is activated, suggesting lectin pathway activation. ${ }^{112,115} \mathrm{CNT}$ functionalization increases the extension of complement activation, in addition to changing cell uptake. Additionally, the opsonization of CNTs induces the modulation of inflammatory and proinflammatory cytokines by phagocytic cells such as monocytes. ${ }^{73,87}$ Thus, CNTs activate both the classical and alternative complement pathways, with the classical pathway being predominant. The activation of complement $\mathrm{C} 3$ and $\mathrm{C} 5$ indicates the formation of the MAC. ${ }^{116}$ Previous studies have shown that the differences in the activation of the complement system are related to the degree of functionalization of the CNTs and the chemical nature of the substances used in the functionalization of these nanomaterials. ${ }^{111}$

\section{Immunosuppressive Effects of Carbon Nanotubes}

CNTs also have the characteristics of inductions of immunosuppression. Previous studies have shown that after exposure to $1 \mathrm{mg} / \mathrm{m} \mathrm{MWCNTs}$ for 14 days, no inflammation or tissue damage was observed in the lungs. However, the mRNA levels that encode the immunoregulatory cytokine IL-10 were increased, inducing systemic immunosuppression as demonstrated by reduced spleen T cell proliferation. ${ }^{117}$
Other studies investigated the levels of TGF- $\beta$ released by alveolar macrophages after phagocytosis of MWCNTs. TGF- $\beta$ is an anti-inflammatory cytokine secreted by phagocytic cells to avoid unwanted immune responses. ${ }^{118}$ These events lead to the activation of the cyclooxygenase $(\mathrm{COX})$ route, inducing the production of prostaglandin and IL-10 and altering systemic immunity. Other mechanisms of immunosuppression induced by CNTs have shown that pharyngeal aspiration of acid-treated CNTs in mice induces a weaker systemic response of $\mathrm{T}$ cells and DCs, which is responsible for this suppression. ${ }^{119}$

\section{Relations Between Physicochemical and Immunomodulatory Properties of Carbon Nanotubes}

The immune response to CNTs can be orchestrated/modulated according to the physicochemical properties of these nanomaterials that influence not only the mechanisms related to cellular uptake but also the induction of specific signaling pathways. ${ }^{72}$ Thus, modifying the physicochemical properties of CNTs, such as changing their length and surface charge, affects their uptake by cells and, consequently, the targeting and potency of the immune response induced by this modification.

Hassan et al modified the physicochemical properties of CNTs to subsequently conjugate with the antigen OVA. Long CNTs conjugated with OVA (+- $386 \mathrm{~nm}$ and a positive charge of $5.8 \mathrm{mV}$ ) were compared with short CNTs conjugated with OVA (+- $122 \mathrm{~nm}$ and a negative charge of -23.4 or $-39 \mathrm{mV}$ ). Compared with long nanotubes, short CNTs showed better uptake by cells and a better immune response both in vitro and in vivo. The reduction of the negative charge of the nanotubes promoted better cellular uptake and, consequently, increased the intensity of the immune response. These results demonstrate the potential of CNTs as a basis for immunotherapies/vaccines. ${ }^{60,120}$

The physical-chemical characteristics of nanotubes have a direct correlation with their immunomodulatory activity. Studies performed by Bianco et al demonstrated that the immune response induced by the CNT CpG-ODN + lysine conjugate is more robust than that induced by the CNT CpG-ODN+ammonia conjugate. Consequently, the immune response activation induced by the physicochemical properties of nanotubes is directly related to increased cellular uptake. ${ }^{121}$ Modification/functionalization with positively charged 1.3-dipolar cycloaddition of azomethine 
ylides significantly improves the uptake of nanotubes by cells. A previous study compared four different types of CNTs functionalized with the following characteristics in epithelial and monocyte cell cultures: i) 1.3-dipolar (12.95 $\mathrm{mV})$, ii) oxidized $(-52.61 \mathrm{mV})$, iii) amidated $(-2.35 \mathrm{mV})$ and iv) polyetherimide $(53.33 \mathrm{mV})$. A direct correlation between the surface chemical modifications of CNTs and their cellular uptake was shown. ${ }^{60}$

Certain physicochemical modifications of CNTs have a significant impact on the development of pulmonary fibrosis. ${ }^{118}$ Anionic functionalization $(\mathrm{COOOH}$ and $\mathrm{PEG})$ of nanotubes decreases the production of profibrinogenic cytokines and growth factors, especially IL-1B and TGF-B1, while neutral or cationic functionalization $(\mathrm{NH} 2$ and sw-NH2) of nanotubes yields intermediate effects. These differences may be related not only to the cellular uptake of the nanotubes but also to the activation of the NLRP3 inflammasome in macrophages, which is dependent on cathepsin B induction. ${ }^{122}$

Monocytes and $\mathrm{T}$ cells, upon uptake/internalization of oxidized CNTs (Ox-MWCNT-2), show downregulation of ribosomal protein-associated genes. The most relevant identified signaling pathways include the induction of IL6, CD40 DC maturation, TNFa/TNFR1-2 and NFkB signaling. ${ }^{73,87}$ Physicochemical modification of CNTs not only alters but also directs the immune response, which can be activated and intensified according to the treatment of this nanomaterial. Thus, cationic functionalization is crucial when the goal is biological/biomedical applications. Cationic CNTs induce the production of cytokines and growth factors, as well as the synthesis of IL-1B, PDGF-AA and TGF-B1, which confirms the importance of physicochemical modification of these nanomaterials for the induction of a specific immune response. ${ }^{122}$

The results obtained using nanotechnological strategies provide insights into the immunomodulation that changes according to the different physicochemical properties of CNTs, demonstrating the potential for the application of these nanomaterials as immunotherapeutic agents and/or vaccine adjuvants.

\section{Perspectives on Vaccine Adjuvants and Carbon Nanotubes Use Commonly Used Adjuvants and Adjuvants with Increased Immunological Potential}

The aim of a vaccination is to induce an immune response that confers long-term protection against infection. However, the efficacy of a vaccine is also related to the additional use of adjuvants, which have immuneenhancing properties that promote increased efficacy and timing of antigen-specific immune responses, especially T cell responses. ${ }^{4,16}$

Adjuvants are generally classified into two categories: vehicles (eg, mineral salts, emulsions, liposomes), which present vaccine antigens more effectively to the immune system to increase the specificity of the immune response, and immunostimulants (eg, TLR agonists, saponins, cytokines), which affect the immune system by increasing immune responses to antigens through the induction of cytokines and costimulatory signals. ${ }^{123}$

Despite the progress made in the use of adjuvants, drug regulatory agencies are searching for new adjuvants because they are not always strong enough to culminate in a long-lasting protective response against the target pathogen. Until recently, the focus of vaccine studies/ development has been on traditional adjuvants such as calcium and aluminum salts. However, new regulatory approaches for vaccine adjuvants for use in infectious diseases are being considered by the Center for Biologics Evaluation and Research (CBER) at the FDA, where the number of preinvestigation drug applications has increased considerably in recent years. ${ }^{124}$

Adjuvants act through different mechanisms, eg, induction of long-lived antigen and $\mathrm{CD} 8 / \mathrm{CD} 4$ responses, increase in the time of immune presentation of vaccine antigens by DCs, and targeting of humoral immune responses with specific antibodies. ${ }^{125}$ However, one of the challenges of traditional adjuvants is their safety, as local adverse reactions such as muscle pain, swelling, granulomas, ulcers, and toxicity are some of the unwanted side-effects, as well as systemic reactions that can occur, eg, nausea, fever, arthritis, eosinophilia, immunosuppression, and anaphylaxis. ${ }^{126}$

Although several problems may be associated with the development of vaccine adjuvants, numerous advances have been made in understanding the molecular/cellular basis involved in responses to these compounds. ${ }^{39,127}$ Thus, knowledge of the action of adjuvants is fundamental in the formulation of new vaccines against emerging diseases, eg, HIV, influenza and coronavirus. The development of new formulations that can potentiate the immune response is urgent and necessary, and effective adjuvant systems are being studied for their use in vaccines that require synergy between immunostimulants and the delivery system. ${ }^{15,126}$

Although different types of adjuvants are used in vaccine production, the most common and licensed for use in 
human vaccines are alum (composed of aluminum salts, which increase immunogenicity and antigen stability); emulsion/squalane/MF59 (composed of a biodegradable oil, which induces a stronger antibody response); liposomes (composed of cholesterol/lipids that encapsulate antigens and act as a carrier adjuvant, which induces cytokine and TNF-a production); and saponins (derived from Quillaja saponaria, a Chilean tree that induces cytokine production and interacts with cell membrane cholesterol). ${ }^{127,128}$

Reference vaccine adjuvants, such as alum and MF59, have been the most popular in recent decades and are used in licensed vaccines. The potency of these adjuvants is related to gene activation, which culminates in immunological potency. In this regards, alum is the gold standard in the formulation of potent vaccines due to its wide availability, familiar mechanisms of action, and low cost. Alum acts by inducing, for example, the release of uric acid and ATP, which recruits inflammatory cells into the muscle. $^{125,128}$ MF59 has effects on proinflammatory genes and in the recruitment of CD11, in addition to promoting the migration/differentiation of DCs. Studies have shown that both alum and MF59 act by similar mechanisms regarding the modulation/activation of the immune system. $^{129}$

The H5N1 influenza vaccine, using AS03, induces cross-antibody production by mechanisms involving recruitment of preexisting cross-memory $B$ cells and activation of naïve $B$ cells, which may be the key to nextgeneration influenza vaccines. GSK Company has used this approach in adjuvant system development, which has culminated in the most successful vaccine adjuvant to date. $^{130}$

One of the adjuvants with the greatest potential is the association of alum-MPL with MF59 (AS04), which is currently used in vaccine systems that have been administered to thousands of people. Several advances are being made to associate well-established adjuvants together with new compounds/materials whose formulations represent the next generation of vaccines with well-understood mechanisms of action that can be produced on a large scale. $^{123}$

The development of new vaccine systems using more potent adjuvants is urgent and necessary in conferring disease protection, especially emerging infections such as HIV, influenza and coronavirus. From this perspective, the possibility of developing strategies that address wellestablished adjuvants and their association with nanomaterials represent possibilities to be considered in the modulation/orchestration of an efficient immune system. $^{23,39}$

\section{Carbon Nanotubes as Vaccine Scaffolds}

Vaccines are biological products used in immunization processes to produce antibodies against diseases caused by pathogenic microorganisms. ${ }^{131}$ However, some of the most significant problems include inadequate adsorption, anaphylactic reactions and hypersensitivity caused by vaccine adjuvants or antigens. Therefore, the development of new materials to improve vaccines has recently been of great interest. ${ }^{126}$ The potency of a vaccine lies in its ability to induce a comprehensive immunological response at the cellular level, as well as stimulate T cells and the destruction of infected cells through the production of neutralizing antibodies promoting opsonization and, consequently, pathogen clearance. ${ }^{8}$

CNTs are potential candidates as an element of vaccines, especially as a function of molecular transport, called the carrier effect. It is possible to couple specific antigens, which increases the interaction with the immune system. CNTs, when functionalized, are relatively inert and not immunogenic or toxic per se. ${ }^{10,60} \mathrm{CNTs}$, due to their unique characteristics, including a surface composed solely of carbon atoms, make it possible to efficiently couple different molecules, including antigens/immunogens, on their surface. In addition, CNTs are insoluble, which prolongs their effects on immune response activation. $^{100}$

CNTs can be functionalized and engineered with specific particles, whose objective is to enhance the immune response, increasing its specificity, stability and immune efficiency. Commonly, vaccines use adjuvant formulations to enhance immunological effects, but the use of CNTs does not require the use of these formulations, since these nanomaterials have intrinsic adjuvant properties. ${ }^{132}$ The use of CNTs in the development of nanovaccines represents the "holy grail" in the battle against different diseases, including infections with viruses such as HIV and SARS-CoV-2. ${ }^{26,29,55}$ Therefore, investigating CNT applications related to the immune system is essential to guide the development of nanovaccine research. ${ }^{106}$

Depending on their size and type of functionalization, CNTs have the potential to activate immune system cells, including monocytes, macrophages and DCs, which are essential to induce the innate immune response. DCs process peptides embedded in CNT walls and present them on 
the main histocompatibility complex molecules MHC class I and II, culminating in both cellular and humoral immune responses. ${ }^{99}$

CNTs associated with epitopes/peptides for B-cell epitopes of foot-and-mouth disease virus (FMDV) or those associated with the N-terminal residue of the apical membrane of $P$. vivax, a malaria agent, induce high antibody titers. Additionally, animals immunized with Plasmodium are protected against challenge with species of Plasmodium. $^{22}$ Initial attempts to apply CNTs as a supporting tool for vaccine development involved the covalent attachment of peptides from the FMDV viral envelope to CNTs. ${ }^{133}$ This immune response was more robust than that to the free peptide, confirming the adjuvant effect of CNTs. These nanotechnological systems, called nanoplatforms, are composed of CNTs + peptides and showed that it was possible to retain the epitope structure in an immunogenic way when connected to CNTs. $^{133}$

Cancer treatment using CNTs is an emerging area with good prospects for immunotherapeutic development. ${ }^{8}$ Molecular complexes of the CNT viral protein are capable of generating specific immune responses in animal models. Pantarotto et al showed that only the CNT peptide elicited neutralizing IgG responses. ${ }^{133}$ Meng et al used a tumor cell lysate conjugated to CNTs as a cancer treatment in a murine hepatoma model. The conjugate vaccine improved cure rates compared with lysates alone, apparently by enhanced activation of cytolytic T cells. ${ }^{134}$

The purified protein derivative of tuberculin conjugated to a nanotube/CNT-PPD antigen was used to study the characteristics of $\mathrm{T}$ cell responses in rats. Interestingly, while traditional adjuvants, such as PPD in Freund's adjuvant, generated a predominantly Th-2 response, the CNTPPD response was skewed toward a Th-1, interferon and IL-12 cytokine response. ${ }^{135}$

Mocan et al compared the effects of CNTs and embryonic stem cells injected separately but in the same murine models to suppress murine colon carcinoma growth. This combination was more effective than any agent administered alone, and in both cases, the activation of CD8 lymphocytes was improved. ${ }^{59}$ Wilm's tumor protein (WT1) is overexpressed in many human leukemias and cancers and is widely used in clinical trials as a cancer vaccine. ${ }^{136}$ Mice immunized with the CNT peptide vaccine and adjuvant induced specific IgG responses against the peptide. The peptide CNT vaccine was internalized in DCs and macrophages in vitro, promoting immunization.
The peptide alone with an adjuvant did not induce an immune response in mice. ${ }^{98}$

\section{Functionalization of Carbon Nanotubes Aimed at Immunostimulation or Nanoplatforms}

CNTs have the ability to link multiple copies of antigens or immunological stimulants simultaneously, which allows the design of new approaches for their use in vaccine construction. ${ }^{8}$ Different studies have shown that cellular damage is minimized by CNT-f compared with nonfunctionalized CNTs (ie, PCNTs). ${ }^{137}$ When chemical modification occurs on the surface, the cellular viability of CNTs improves biocompatibility, especially when using functionalization methods such as 1.3-dipolar cycling, oxidation/ amidation or acid treatment. ${ }^{77}$

Thus, the resulting cellular damage varies depending on the surface load generated in the different functionalization methods as well as the nature of the coupled molecules. Previous studies have reported that PEG coating (PEGylation) reduces the interaction of NPs with immune receptors. ${ }^{138}$ Unlike anionic functionalization (PEG and carboxylic groups), cationic functionalization, such as polyethyleneimine, favors cell uptake and, consequently, the release of cathepsin $\mathrm{B}$ peptidase, culminating in the activation of the NLRP3 inflammasome. ${ }^{122}$

Inflammasomes are multiprotein complexes (important modulators of chronic inflammation) expressed and formed mainly in phagocytic cells after hazard detection, modulating the secretion of proinflammatory cytokines such as IL- $1 \beta$ and IL-18 through caspase- 1 activation. In general, the metallic contaminants present in CNTs are responsible for the activation of the NLRP3 inflammasome. Additionally, exogenous signals such as microbial molecules or aluminum salts and endogenous signals such as heat shock proteins or adenosine-ATP triphosphate activate the NLRP3 inflammasome. ${ }^{87}$

CNT-f, which is modified on its surface, has drastically reduced levels of toxicity. ${ }^{77}$ Thus, CNT-f with 1.3-dipolar prevents the activation of inflammatory cytokines such as IL-6 and TNF- $\alpha{ }^{139}$ CNT-f with ammonium induces modulation of immunoregulatory pathways without expressing apoptosis pathways, and when oxidized, these nanomaterials do not cause a cytotoxic effect. In this way, the blockade of inflammatory pathways indicates that CNT-f is a promising nanostructure for different biomedical and biological applications. $^{140}$ 
CNTs are considered successful nanomaterials in the development of nanostructural constructs, such as nanoplatforms that can transport antigens (the carrier effect) and stimulate the synthesis of $\mathrm{T}$ lymphocytes, which are central immune cells in the initiation and maintenance of immune responses. ${ }^{10,60}$ Generally, T lymphocytes are targeted for therapies, especially vaccination, in which the immune system needs to be activated. Nanocarriernanoplatform systems can increase the immunogenicity of a molecule. Previous studies have shown that chitosan NPs conjugated to H1N1 and nanoplatforms containing gold-coated Yersinia pestis F1 antigen (AuNPs) produced higher levels of antibodies and cytokines than the administration of unconjugated antigens. ${ }^{141}$

Bundles of CNTs carrying molecules capable of activating $\mathrm{T}$ lymphocytes, such as specific antibodies specific for CD3 and CD28 molecules, mimic T cell physiological activation by APCs. These studies showed that CNT-f that was treated to generate hydroxyl terminal groups $\left(\mathrm{HNO}_{3}\right.$ $\left./ \mathrm{LiBH}_{4}\right)$ increased antibody adsorption. These results clearly show that CNT-f nanoplatforms have potent effects on $\mathrm{T}$ cell activation. ${ }^{142}$ These nanoplatforms increased the magnitude and kinetics of the immune response compared with those observed with the soluble form of antibodies or other substrates with a high surface area, such as polystyrene beads or C60 NPs. The use of CNTs as substrates allowed an increase in the magnitude of the response of antigen-specific $\mathrm{T}$ cells compared with soluble controls only. This nanoplatform antigen presentation system proved efficient since $T$ cells interacted specifically with this artificial antigen presentation platform. ${ }^{18}$

\section{Adjuvant Effect of Carbon Nanotubes}

One vaccine component is the adjuvant, a substance with immunogenic potential that is part of the immunization strategy but does not induce an immune response per se. CNTs have inherent adjuvant properties that stimulate the immune system. ${ }^{132}$ Previous studies have shown that the therapeutic effect on the formation of colon cancer in a C57BL/6 animal model changes when CNTs are administered as an adjuvant in embryonic cells, which promotes a decrease in tumor volume and an increase in the cytotoxicity of CD8+ cells, Th1 and IFN cytokines and IL2 , inducing an immunosuppressive effect. ${ }^{59}$

CNTs can be conjugated to unmethylated DNA $+\mathrm{CpG}$, which can be used as an adjuvant in vaccines. $\mathrm{CpG}+$ CNTs improve immunostimulatory properties and compensate for the negative charge of $\mathrm{CpG}$, which facilitates its entry into the cell. ${ }^{143}$ Other cells, such as natural killer (NK) cells, macrophages and microglia, quickly internalize CNTs by more than $50 \%{ }^{73}$ New approaches to achieve efficient vaccination are still needed, and CNTs are considered potentially promising, with the aim of transporting antigen/nanoplatforms. Few studies have considered this approach, but compared with classical vaccine protocols, it elicits an improved immune response. ${ }^{10,60}$

The type of immune response induced by a vaccine is a crucial element in eliminating a specific pathogen. Zeinali et al described the ability of a protein derivative from tuberculin to generate a necessary $\mathrm{T}$ cell immune response to achieve tuberculosis protection, which occurred only when this protein was coupled to CNTs. ${ }^{135}$ Additionally, the administration of tumor proteins conjugated to CNTs demonstrated an increase in the effectiveness of a tumor cell vaccine by activating a specific cellular immune response to the tumor. ${ }^{98}$

\section{Physicochemical Characteristics of Carbon Nanotubes That Limit Their Application in Vaccines Physicochemical Properties of Carbon Nanotubes That Alter the Immune \\ Response}

Different characteristics of CNTs, such as the number of walls, diameter, length and type of CNT functionalization, directly influence their cellular uptake and toxicity. In general, interactions of CNTs are noncovalent, resulting in rapid dissociation of nanocarrier antigens. These processes are dependent on the properties of the external environment, such as $\mathrm{pH}$, ionic strength, and temperature, and on the hydrophobicity of the antigen. $^{60}$

\section{Size and Geometry}

Nanoformulations improve the delivery and presentation of antigens, since NP shape, size and surface are key factors directly affecting NP circulation, biodistribution, bioavailability and specificity. In addition to these factors, the particle geometry related to the surface and the volume ratio play a fundamental role in determining the kinetics of immunogen release and degradation. ${ }^{57}$ The size of the NPs is a factor to be considered, since it is related to the mechanism of cellular uptake, immunogenicity and specificity (Figure 7$).{ }^{65}$ Large NPs (1 to $17 \mu \mathrm{m}$ ) 


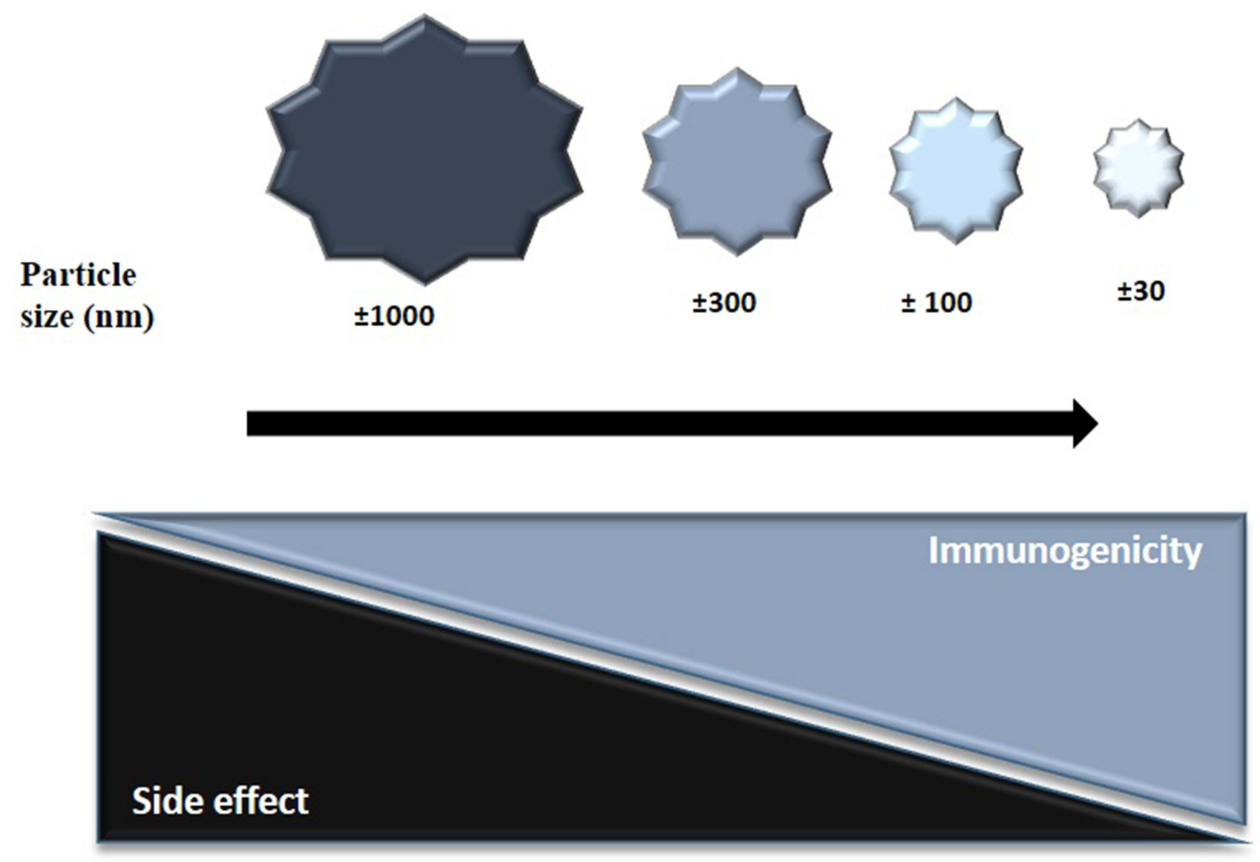

Figure 7 Effect of particle size on immunogenicity and side-effects. Particles smaller than $100 \mathrm{~nm}$ in diameter enter the lymphatic system effectively compared to particles larger than $300 \mathrm{~nm}$ in diameter. Particles smaller than $30 \mathrm{~nm}$ in diameter induce a stronger CD8 T cell response, compared to particles larger than I00 nm that promote a stronger CD4 T cell response. The strength of the T cell response as well as the side-effects correlate inversely with particle size.

have a reduced internalization rate compared with that of smaller NPs $( \pm 300 \mathrm{~nm})$. The size of the NPs also determines the cellular capacity for specificity and migration. Smaller NPs ( $\pm 20-200 \mathrm{~nm})$ are transported and endocytosed by DCs and subsequently drained into the lymph nodes, while larger NPs $( \pm 2.000 \mathrm{~nm})$ are absorbed by migratory DCs. ${ }^{65,72}$

Activation of signaling pathways is also affected by the size of the NPs, with smaller NPs altering cell signaling processes more efficiently than large NPs. In this sense, NPs of $\pm 150 \mathrm{~nm}$ in diameter and $\pm 450 \mathrm{~nm}$ in height showed greater cell uptake than particles with a size of $1.200 \times 200 \mathrm{~nm}$. Another feature is the geometry of the NPs, as the curvature of the NPs directly affects cell interactions and the rate of phagocytosis. ${ }^{72,96,144}$

\section{Hydrophobicity}

The hydrophobicity of NPs plays a fundamental role in the interaction with soluble proteins and immune cells through the recognition of hydrophobic portions. Similar observations have been reported for other innate immune cells, as hydrophobic NPs were able to activate different cell types, upregulate proinflammatory cytokine expression, and encode genes that facilitate the opsonization process, increasing the adsorption of immunoglobulins on the cell surface. ${ }^{15,145}$

\section{Advantages, Disadvantages and Applications of the Different Types of Carbon Nanotubes}

CNTs differ in both structure and physicochemical aspects. In biological/biomedical applications, the most commonly used CNTs are SWCNTs and MWCNTs. ${ }^{56,80}$ Importantly, the differences between CNTs are related primarily to physicochemical modifications/functionalizations, which enable the efficient and safe use of these nanomaterials. Therefore, the physical-chemical characteristics of nanotubes, eg, degree of purity, size and functionalization, are critical factors that lead to different reactions on cells.

One of the greatest advantages of using CNTs is that they are extremely small, with excellent moldability, temperature resistance and electrical conductivity. ${ }^{56,146}$ Another difference between nanotubes to be considered is related to the synthesis processes of this nanomaterial, which can occur by different methods, including the chemical vapor deposition (CVD) technique, laser ablation and arc discharge. ${ }^{137,147}$

The main advantage of the arc-discharge technique is the potential to produce CNTs in large quantities, and a disadvantage of this synthesis method is the small control related to the alignment/chirality of these nanomaterials, which affects their use in biological applications. In the laser alloying technique, the main advantage is the 
yield and reduction of metallic impurities, since the metallic atoms involved tend to evaporate from the end of the tube when it is closed. ${ }^{148}$ The main disadvantage of the laser ablation technique is that nonuniform nanotubes are obtained, usually with some branching. Laser ablation and CVD have advantages since they allow the production of purer nanotubes (fundamental for biological/medical applications), and are economically more feasible for the large-scale production of CNTs. ${ }^{148,149}$

To be integrated into biological systems, CNTs need to undergo physical/chemical modifications, including coupling with therapeutically active molecules, which will enable the targeting of these nanomaterials for immunotherapies, imaging and/or specific diagnostics. ${ }^{57,} 78$ A common difference between CNTs lies in their structural characteristics. Accordingly, Saleh et al analyzed CNTs of different subtypes, namely, straight and tangled nanotubes. In this study, intracellular/intrapulmonary spraying was performed in rats, and it was observed that straight CNTs, similar to asbestos fibers, caused lung carcinogenesis when administered via the airway and, when administered via the peritoneal cavity, caused mesothelioma. However, entangled nanotubes administered into the peritoneal cavity did not induce carcinogenesis. ${ }^{150}$

Another difference related to the physicochemical modification of CNTs is the anionic functionalization (carboxylated and PEGylated) of these nanomaterials. Anionic CNTs are associated with decreased production of cytokines and growth factors by THP1 cells (IL-1beta) or by THP1 cells in bronchial epithelial cell lines (PDGF-AA and TGF-beta). ${ }^{122}$ These carboxylated nanotubes did not induce an inflammatory response; however, the high degree of cationic functionalization (PEI) led to increased cytokine activation associated with the development of lung fibrosis. ${ }^{151}$

Therefore, different types of nanotubes obtained by different synthesis methods and different types of functionalization interfere and drastically alter the cellular recognition profile and, consequently, the mechanisms of processing and presentation of antigens to the cells. In phagocytes, nanotube functionalization with anionic phospholipids (phosphatidylserine and diacylpipatidylglycerol) drastically alters the recognition and uptake of these nanomaterials. CNTs produced by the HiPCO technique induce lung inflammation. This type of nanotube not only facilitates the recruitment of DCs into the lungs but also interferes with the activation of DCs. ${ }^{99}$

A comparative study analyzed the induction of carcinogenicity according to different types of CNTs. Four
CNTs were analyzed (named M-, N-, WL-, and SD1CNTs), with different structural features, including circular, straight, and long fibers; these CNTs were $5 \mu \mathrm{m}$ in length and $60 \mathrm{~nm}$ in thickness and had the characteristic of forming few clusters. CNTs with these structural features induced mesothelioma at a rate of $100 \%$. CNTs with long and thick structures are more likely to induce mesothelioma because they escape from the lymphatic drainage system. ${ }^{152}$ These CNTs undergo frustrated phagocytosis and are associated with the expression of cytokines and ROS, which constitute one of the major mechanisms of carcinogenicity. Three other types of CNTs (named WS-, SD2- and T-CNTs), with a coiled/compounded shape, short length $(<3 \mu \mathrm{m})$, thin wall $(<50 \mathrm{~nm})$, and the ability to form clusters, were also analyzed in this study. It was observed that these structurally different CNTs did not induce mesothelioma. Thus, the circular or straight shape of CNTs might be critical for the formation of carcinomas/ mesothelioma. ${ }^{152}$

The structural characteristics of nanotubes are critical factors in mesothelioma and carcinogenesis induction, and the shape, size and diameter of these nanomaterials are critical factors in this process. ${ }^{64,72}$ Importantly, CNTs less than $1 \mu \mathrm{m}$ in length and with a functionalized surface decrease the risk of adverse reactions and the induction of toxicity. ${ }^{72}$ However, despite the advances and advantages of using CNTs, one of the biggest concerns is related to their toxicity, attributed especially to the physicalstructural similarity between CNTs and asbestos fibers. ${ }^{64}$

CNTS toxicity is strongly related to their size, where nanotubes $>100 \mathrm{~nm}$ have a higher potential for lung toxicity. ${ }^{72}$ Moreover, CNTs can cross biological barriers and accumulate in organs, inducing inflammation and fibrotic reactions. Additionally, the insolubility and the presence of morphological nonuniformity related to the synthesis/purification processes are factors that represent disadvantages in the use of these nanomaterials. ${ }^{153}$ CNTs, when not purified and/or unfunctionalized, commonly form aggregates. These issues, if not addressed, especially regarding the choice of treatment/functionalization of the nanotube, represent major problems, making its use in biological/medical systems unfeasible. ${ }^{77,139}$

In this context, purification of CNTs after their synthesis is essential for their use in biological/biomedical applications. The goal of purification of nanotubes is to eliminate/ reduce the compounds resulting from their synthesis, including the elimination of large graphite particles and amorphous aggregates, through dissolution in solvents to eliminate 
catalyst particles and fullerenes. ${ }^{153}$ Common techniques in the purification process of CNTs consist of microfiltration or chromatography to separate the particles by size and remove amorphous carbon clusters. ${ }^{154}$

Also, a challenging issue in the use of CNTs is related mainly to their biodistribution and pharmacokinetics, which can be altered according to differences in the chemical composition, aggregation, and surface/morphology of these nanomaterials. ${ }^{155}$ However, these limitations can be overcome by the process of functionalization/physicalchemical modification through the conjugation of therapeutic molecules or ligands to make them active and allow unprecedented advances in the treatment of various pathologies against diseased and/or cancer cells. ${ }^{156}$

With the advances made to date, it is clear that CNTs, which have been widely explored, have significant potential for a wide range of biological/biomedical applications. Therefore, the development of new approaches to the physicochemical structures of CNTs will strategically drive the visionary use of nanobiotechnologies and, in the near future, will optimize approaches that leverage new levels of potential biological applications. ${ }^{157}$

\section{Challenges in Using Carbon Nanotubes as Vaccine Agents}

\section{Aspects of Toxicity and Functionalization}

Nanomaterials have unique characteristics, and their interactions can have significant biological impacts. For years, CNTs have been considered hazardous materials, like asbestos fibers, with important toxicological effects, especially the induction of lung toxicity. ${ }^{158}$ Nonfunctionalized CNTs (ie, PCNTs), act as foreign bodies in the cell, and the immune response against that foreign body is triggered, culminating in the production of oxidative processes to eliminate CNTs from the cell. ${ }^{159}$

PCNTs have been associated with high levels of inflammation, granuloma and upper airway obstruction in a murine model. In contrast, CNTs functionalized via different methods (PEGylation, amino acid modification, etc.), low inflammation levels are observed. ${ }^{119,138}$ PCNTs induce secretion of IL-1 beta linked to caspase-1, which is highly cytotoxic. These mechanisms can mediate the production of several inflammatory cytokines by activating pathways associated with oxidative stress and the caspase cascade. $^{58,95}$

PCNTs induce oxidative stress in cells, with the production of protein kinases and nuclear factor kappa B, and these factors are key to the regulatory signaling of cytokines in response to the oxidative stress generated by these nanomaterials. The cellular toxicity generated by PCNTs occurs through the generation of free radicals, leading to oxidative stress. ${ }^{158,159}$ Accordingly, excess free radicals oxidize DNA, proteins and lipids in cells through the activation of transcription factors by activating protein-1, which is responsible for the inflammatory response. ${ }^{160}$

CNTs cannot be easily eliminated from the body. They have a high risk of accumulating in organs such as the spleen, kidneys and lungs, which are generally targets for oxidative stress caused by the formation of free radicals and the induction of toxicity by the generation and accumulation of ROS. ${ }^{64,159}$

PCNTs can bind to various cytoplasmic proteins and activate the innate immune response and the complement pathway, leading to inflammation. ${ }^{113,115}$ Despite their adjuvant effect, they can have a harmful impact on the host if high inflammation levels are induced. Thus, one of the biggest concerns related to the use of CNTs as vaccine structures or other therapeutic-prophylactic proposals is the risk of bioaccumulation and toxicity. ${ }^{24,161}$

However, their small size makes it possible for them to spread throughout the body and reach crucial sites, an ability that may be either beneficial or harmful in vaccineimmunotherapeutic processes, depending on the design of the nanostructure. To prevent or reduce toxicity, CNTs must be functionalized to make them more soluble in water, more biocompatible, and, consequently, less toxic. ${ }^{78} \mathrm{CNT}-\mathrm{f}$ is biocompatible and can be eliminated in urine and feces. ${ }^{155}$ The biocompatibility of CNT-f makes them less toxic, avoiding the effects of clustered accumulation of these nanomaterials in organs. To decrease toxicity, purification of CNTs is a fundamental step, which allows the removal of toxic chemical products or even the metals used during the production of CNTs. ${ }^{162}$

\section{Discussion and Prospects Regarding the Use of Carbon Nanotubes as Nanovaccines}

Immunological adjuvants are essential to induce a robust immune response. ${ }^{15}$ Nanotechnological vaccines are promising for improving the immunogenicity of a vaccine antigen, offering advantages over other traditional adjuvant approaches, including enhanced stability, prolongedrelease kinetics, reduced immunotoxicity and improved immunogenic selectivity. ${ }^{12,13}$ 
One of the most significant advantages of nanotechnological vaccines based on CNTs is the possibility of combining several active and specific molecules to achieve the desired immune activation. ${ }^{98,141}$ CNTs are widely investigated nanomaterials that transport specific molecules into cells of the immune system. In this way, decreasing the toxicity and increasing the biocompatibility of these nanomaterials used to transport therapeutic molecules remain significant challenges to overcome. ${ }^{77}$

Typically, vaccines use adjuvants, aiming to enhance the immunogenic effect without inducing an immune response per se; in contrast, CNTs do not require adjuvants, since they have intrinsic adjuvant properties. ${ }^{132} \mathrm{CNTs}$, independent of their conjugated to specific biomolecules, have the potential to orchestrate the immune system, especially $\mathrm{T}$ cells. ${ }^{98,107}$ Most studies on CNTs are related to their toxicity and biocompatibility, and few provided insights into their effects on immune cells. However, several studies related to nanotechnological vaccines based on CNTs have demonstrated that the vaccine antigen's immunogenicity is increased, as the use of CNTs enables both carrier effects and immunomodulatory activity. ${ }^{39}$

Vaccines based on CNTs are also exciting due to their possibility of modifying the surface of the nanomaterial with specific molecules of high affinity, which can significantly increase the immune response generated by the specific, effective and stable release of an antigen. ${ }^{60}$

Nanotechnological delivery systems make it possible to improve the humoral and cellular immune response due to their nanoscale size, which facilitates uptake by phagocytic cells, inducing specificity and efficiency in antigen recognition and presentation. ${ }^{10,60}$ The possibilities for CNT action and use are diverse, and expectations related to their biomedical and biological applications are high; indeed, this is a topic of great interest to the scientific and industrial communities. ${ }^{3,156}$ However, the potential use of nanomaterials in products requires urgent knowledge regarding their safety, toxicity, biocompatibility and biodegradability. ${ }^{77}$

\section{Conclusions}

Nanovaccines represent one of the most powerful resources to promote the immunogenicity of a specific antigen, with several advantages over other vaccine approaches including: longer time to presentation/extended stability of the antigen; more robust and durable immune response; decreased toxicity as well as the ability to couple multiple immunogenic molecules, factors that represent unprecedented advantages when compared to traditional vaccines. The tools of nanotechnology provide technological strategies with unprecedented possibilities in the treatment/cure of several diseases, infectious or not. In this sense, the use of carbon nanotubes as candidates in vaccine systems, present good results when functionalized, since they are inert, nonimmunogenic or toxic per se making possible the efficient coupling of different molecules simultaneously. However, one of the major concerns of the use of CNTs is the toxicity of these nanomaterials, mainly related to physicochemical and structural aspects that, if not addressed, as far as functionalization issues are concerned, can make their use in biological/medical systems unfeasible. Thus, investigating the use of CNTs as a state-of-the-art approach to target the immune system will facilitate the development of "safedesign" nanovaccines based on CNTs to treat different infections. Knowledge regarding how to adapt and modify the physical and chemical properties of CNTs for use in nanovaccines and immunotherapies to improve their effectiveness in inducing a $\mathrm{T}$ cell-based immune response has become an urgent need in the defense against cancer and different infections, including infections with viruses such as HIV and coronavirus.

\section{Declaration on This Paper}

The abstract of this paper was presented at the Webinar on Microbial Biotechnology and Gene Therapy on 29th January 2021 at 12:10 PM as a commentary-lecture presentation with interim findings. The abstract was published in Poster Abstracts in "Research \& Reviews: Journal of Social Sciences.”

\section{Funding}

This work was financially supported by the 2012/1142283 grants from the Coordenação de Aperfeiçoamento de Pessoal de Nível Superior and by the Ministério da Saúde MS-SCTIE-Decit/CNP no.12/2018. The funders had no role in study design, data collection or analysis, the decision to publish or the preparation of the manuscript.

\section{Disclosure}

The authors have no conflicts of interest associated with this work.

\section{References}

1. Jaison JJ. Review on nanoparticles and nanostructured materials: history, sources, toxicity and regulations. Beilstein J Nanotechnol;2018;9 (1):1050. doi:10.3762/bjnano.9.98 
2. Dacoba TG, Olivera A, Torres D, Crecente-Campo J, Alonso MJ. Modulating the immune system through nanotechnology. Semin Immunol. 2017;34:78-102. doi:10.10 16/j.smim.2017.09.007

3. Huang Y, Zeng J. Recent development and applications of nanomaterials for cancer immunotherapy. Nanotechnol Rev. 2020;9 (1):367-384. doi:10.1515/ntrev-2020-0027

4. Vetter V, Denizer G, Friedland LR, Krishnan J, Shapiro M. Understanding modern-day vaccines: what you need to know. Ann Med. 2018;50(2):110-120. doi:10.1080/07853890.2017.14 07035

5. Pollard AJ, Bijker EM. A guide to vaccinology: from basic principles to new developments. Nat Rev Immunol. 2021;21 (2):83-100. doi:10.1038/s41577-020-00479-7

6. Smith KA. Louis Pasteur, the Father of Immunology? Front Immunol. 2012;3:3. doi:10.3389/fimmu.2012.00068

7. Xu J, Lv J, Zhuang Q, et al. A general strategy towards personalized nanovaccines based on fluoropolymers for post-surgical cancer immunotherapy. Nat Nanotechnol. 2020;15(12):10 43-1052. doi:10.1038/s41565-020-00781-4

8. Zhu G, Mei L, Vishwasrao HD, et al. Intertwining DNA-RNA nanocapsules loaded with tumor neoantigens as synergistic nanovaccines for cancer immunotherapy. Nat Commun. 2017;8 (1):1482. doi:10.1038/s41467-017-01386-7

9. Zhu M, Wang R, Nie G. Applications of nanomaterials as vaccine adjuvants. Hum Vaccin Immunother. 2014;10(9):2761-2774. doi: $10.4161 /$ hv.29589

10. Mueller SN, Tian S, DeSimone JM. Rapid and persistent delivery of antigen by lymph node targeting print nanoparticle vaccine carrier to promote humoral immunity. Mol Pharm. 2015;12 (5):1356-1365. doi:10.1021/mp500589c

11. Liu W-L, Zou M-Z, Liu T, et al. Cytomembrane nanovaccines show therapeutic effects by mimicking tumor cells and antigen presenting cells. Nat Commun. 2019;10(1):3199. doi:10.1038/ s41467-019-11157-1

12. Dölen $\mathrm{Y}$, Valente $\mathrm{M}$, Tagit $\mathrm{O}$, et al. Nanovaccine administration route is critical to obtain pertinent iNKt cell help for robust anti-tumor T and B cell responses. Oncoimmunology. 2020;9 (1):1738813. doi:10.1080/2162402X.2020.1738813

13. Li M, Zhou H, Jiang W, Yang C, Miao H, Wang Y. Nanovaccines integrating endogenous antigens and pathogenic adjuvants elicit potent antitumor immunity. Nano Today. 2020;35:101007. doi:10.1016/j.nantod.2020.101007

14. Bhardwaj P, Bhatia E, Sharma S, Ahamad N, Banerjee R. Advancements in prophylactic and therapeutic nanovaccines. Acta Biomater. 2020;108:1-21. doi:10.1016/j.actbio.2020.03. 020

15. Ni Q, Zhang F, Liu Y, et al. A bi-adjuvant nanovaccine that potentiates immunogenicity of neoantigen for combination immunotherapy of colorectal cancer. Sci $A d v$. 2020;6(12):eaaw6071. doi:10.1126/sciadv.aaw6071

16. Kim CG, Kye Y-C, Yun C-H. The Role of nanovaccine in cross-presentation of antigen-presenting cells for the activation of CD8+ T cell responses. Pharmaceutics. 2019;11(11):612. doi:10.3390/pharmaceutics11110612

17. Karchemski F, Zucker D, Barenholz Y, Regev O. Carbon nanotubes-liposomes conjugate as a platform for drug delivery into cells. J Control Release. 2012;160(2):339-345. doi:10.1016/ j.jconrel.2011.12.037

18. Kim H, Kim EH, Kwak G, Chi S-G, Kim SH, Yang Y. Exosomes: cell-derived nanoplatforms for the delivery of cancer therapeutics. Int J Mol Sci. 2020;22(1):14. doi:10.3390/ijms22010014

19. Heo MB, Lim YT. Programmed nanoparticles for combined immunomodulation, antigen presentation and tracking of immunotherapeutic cells. Biomaterials. 2014;35(1):590-600. doi:10.10 16/j.biomaterials.2013.10.009
20. Nikpoor AR, Jaafari MR, Zamani P, et al. Cell cytotoxicity, immunostimulatory and antitumor effects of lipid content of liposomal delivery platforms in cancer immunotherapies. A comprehensive in-vivo and in-vitro study. Int $J$ Pharm. 2019;567:118492. doi:10.1016/j.ijpharm.2019.118492

21. Rosenkrands I, Vingsbo-Lundberg C, Bundgaard TJ, et al. Enhanced humoral and cell-mediated immune responses after immunization with trivalent influenza vaccine adjuvanted with cationic liposomes. Vaccine. 2011;29(37):6283-6291. doi:10. 1016/j.vaccine.2011.06.040

22. Moon JJ, Suh H, Polhemus ME, Ockenhouse CF, Yadava A, Irvine DJ. Antigen-displaying Lipid-Enveloped PLGA Nanoparticles as Delivery Agents for a Plasmodium vivax Malaria Vaccine. PLoS One. 2012;7(2):e31472. doi:10.1371/journal.pone. 0031472

23. Campos EVR, Pereira AES, de Oliveira JL, et al. How can nanotechnology help to combat COVID-19? Opportunities and urgent need. J Nanobiotechnology. 2020;18(1):125. doi:10.1186/ s12951-020-00685-4

24. Singh L, Kruger HG, Maguire GEM, Govender T, Parboosing R. The role of nanotechnology in the treatment of viral infections. Ther Adv Infect Dis. 2017;4(4):105-131. doi:10.1177/204 9936117713593

25. Lauster D, Klenk S, Ludwig K, et al. Phage capsid nanoparticles with defined ligand arrangement block influenza virus entry. Nat Nanotechnol. 2020;15(5):373-379. doi:10.1038/s41565-0200660-2

26. Vela Ramirez JE, Roychoudhury R, Habte HH, Cho MW, Pohl NLB, Narasimhan B. Carbohydrate-functionalized nanovaccines preserve HIV-1 antigen stability and activate antigen presenting cells. J Biomater Sci Polym Ed. 2014;25(13):1387-1406. doi:10.1080/09205063.2014.940243

27. Hung S-J, Hsu Y-M, Huang S-W, et al. Genetic variations on 31 and 450 residues of influenza A nucleoprotein affect viral replication and translation. J Biomed Sci. 2020;27(1):17. doi:10.1186/ s12929-019-0612-z

28. Allen JD, Ross TM. Next generation methodology for updating HA vaccines against emerging human seasonal influenza A (H3N2) viruses. Sci Rep. 2021;11(1):4554. doi:10.1038/s41598020-79590-7

29. Kanekiyo M, Wei C-J, Yassine HM, et al. Self-assembling influenza nanoparticle vaccines elicit broadly neutralizing H1N1 antibodies. Nature. 2013;499(7456):102-106. doi:10.1038/ nature 12202

30. Deng L, Mohan T, Chang TZ, et al. Double-layered protein nanoparticles induce broad protection against divergent influenza A viruses. Nat Commun. 2018;9(1):359. doi:10.1038/s41467-01702725-4

31. Smith LR, Wodal W, Crowe BA, et al. Preclinical evaluation of vaxfectin adjuvanted vero cell-derived seasonal split and pandemic whole virus influenza vaccines. Hum Vaccin Immunother. 2013;9(6):1333-1345. doi:10.4161/hv.24209

32. Kürsteiner O, Moser C, Lazar H, Durrer P. Inflexal ${ }^{\circledR}$ V-The influenza vaccine with the lowest ovalbumin content. Vaccine. 2006;24(44-46):6632-6635. doi:10.1016/j.vaccine.2006.05.084

33. Klein M, Menta M, Dacoba TG, et al. Advanced nanomedicine characterization by DLS and AF4-UV-MALS: application to a HIV nanovaccine. J Pharm Biomed Anal. 2020;179:113017. doi:10.1016/j.jpba.2019.113017

34. Eisinger RW, Lerner AM, Fauci AS. Human immunodeficiency virus/AIDS in the era of coronavirus disease 2019: a juxtaposition of 2 pandemics. J Infect Dis. 2021. doi:10.1093/infdis/jiab114

35. Fourati S, Flandre P, Calin R, et al. Factors associated with a low HIV reservoir in patients with prolonged suppressive antiretroviral therapy. J Antimicrob Chemother. 2014;69(3):753-756. doi: $10.1093 / \mathrm{jac} / \mathrm{dkt} 428$ 
36. Cuevas JM, Geller R, Garijo R, López-Aldeguer J, Sanjuán R. Extremely High Mutation Rate of HIV-1 In Vivo. PLOS Biol. 2015;13(9):e1002251. doi:10.1371/journal.pbio.1002251

37. Macchione MA, Aristizabal Bedoya D, Figueroa FN, Má M-F, Strumia MC. Nanosystems applied to HIV infection: prevention and treatments. Int J Mol Sci. 2020;21(22):8647. doi:10.3390/ ijms 21228647

38. Trimaille T, Verrier B. Micelle-Based Adjuvants for Subunit Vaccine Delivery. Vaccines. 2015;3(4):803-813. doi:10.3390/ vaccines 3040803

39. Vijayan M. Uthaman, Park. Recent Advances in Nanovaccines Using Biomimetic Immunomodulatory Materials. Pharmaceutics. 2019;11(10):534. doi:10.3390/pharmaceutics11100534

40. Kovochich M, Marsden MD, Zack JA. Activation of Latent HIV Using Drug-Loaded Nanoparticles. PLoS One. 2011;6(4):e18270. doi:10.1371/journal.pone. 0018270

41. Hanson MC, Crespo MP, Abraham W, et al. Nanoparticulate STING agonists are potent lymph node-targeted vaccine adjuvants. J Clin Invest. 2015;125(6):2532-2546. doi:10.1172/JCI79915

42. Crooks ET, Moore PL, Franti M, et al. A comparative immunogenicity study of HIV-1 virus-like particles bearing various forms of envelope proteins, particles bearing no envelope and soluble monomeric gp120. Virology. 2007;366(2):245-262. doi:10.1016/j. virol.2007.04.033

43. Wei X, Zhang G, Ran D, et al. T-cell-mimicking nanoparticles can neutralize HIV infectivity. Adv Mater. 2018;30(45):1802233. doi:10.1002/adma.201802233

44. Cirelli KM, Carnathan DG, Nogal B, et al. Slow delivery immunization enhances hiv neutralizing antibody and germinal center responses via modulation of immunodominance. Cell. 2019;177 (5):1153-1171.e28. doi:10.1016/j.cell.2019.04.012

45. Wahome N, Pfeiffer T, Ambiel I, et al. Conformation-specific display of 4E10 and 2F5 Epitopes on Self-assembling Protein Nanoparticles as a Potential HIV Vaccine. Chem Biol Drug Des. 2012;80(3):349-357. doi:10.1111/j.1747-0285.2012.01423.x

46. Zheng B, Peng W, Guo M, et al. Inhalable nanovaccine with biomimetic coronavirus structure to trigger mucosal immunity of respiratory tract against COVID-19. Chem Eng J. 2021;418:129392. doi:10.1016/j.cej.2021.129392

47. Huang C, Wang Y, Li X, et al. Clinical features of patients infected with 2019 novel coronavirus in Wuhan, China. Lancet. 2020;395(10223):497-506. doi:10.1016/S0140-6736(20)30183-5

48. Lurie N, Keusch GT, Dzau VJ. Urgent lessons from COVID 19: why the world needs a standing, coordinated system and sustainable financing for global research and development. Lancet. 2021;397 (10280):1229-1236. doi:10.1016/S0140-6736(21)00503-1

49. Liu L, Liu Z, Chen H, et al. Subunit Nanovaccine with Potent Cellular and Mucosal Immunity for COVID-19. ACS Appl Bio Mater. 2020;3(9):5633-5638. doi:10.1021/acsabm.0c00668

50. Lin LC, Huang C, Yao B, et al. Viromimetic STING agonistloaded hollow polymeric nanoparticles for safe and effective vaccination against middle east respiratory syndrome coronavirus. Adv Funct Mater. 2019;29(28):1807616. doi:10.1002/adfm.201 807616

51. Coleman CM, Liu YV, Mu H, et al. Purified coronavirus spike protein nanoparticles induce coronavirus neutralizing antibodies in mice. Vaccine. 2014;32(26):3169-3174. doi:10.1016/j.vaccine. 2014.04.016

52. Rana MM. Polymer-based nano-therapies to combat COVID-19 related respiratory injury: progress, prospects, and challenges. J Biomater Sci Polym Ed. 2021;2:1-31. doi:10.1080/09205063. 2021.1909412

53. Cagno V, Andreozzi P, D'Alicarnasso M, et al. Broad-spectrum non-toxic antiviral nanoparticles with a virucidal inhibition mechanism. Nat Mater. 2018;17(2):195-203. doi:10.1038/nmat 5053
54. Raghuwanshi D, Mishra V, Das D, Kaur K, Suresh MR. Dendritic Cell Targeted Chitosan Nanoparticles for Nasal DNA Immunization against SARS CoV Nucleocapsid Protein. $\mathrm{Mol}$ Pharm. 2012;9(4):946-956. doi:10.1021/mp200553x

55. Alimardani V, Abolmaali SS, Tamaddon AM. Recent advances on nanotechnology-based strategies for prevention, diagnosis, and treatment of coronavirus infections. J Nanomater. 2021; 2021:1-20. doi:10.1155/2021/9495126

56. Anzar N, Hasan R, Tyagi M, Yadav N, Narang J. Carbon nanotube - A review on Synthesis, Properties and plethora of applications in the field of biomedical science. Sensors Int. 2020;1:100003. doi:10.1016/j.sintl.2020.100003

57. Bhattacharya K, Mukherjee SP, Gallud A, et al. Biological interactions of carbon-based nanomaterials: from coronation to degradation. Nanomed Nanotechnol Biol Med. 2016;12 (2):333-351. doi:10.1016/j.nano.2015.11.011

58. Orecchioni M, Bedognetti D, Sgarrella F, Marincola FM, Bianco A, Delogu L. Impact of carbon nanotubes and graphene on immune cells. $J$ Transl Med. 2014;12(1):138. doi:10.1186/ 1479-5876-12-138

59. Mocan T, Iancu C. Effective colon cancer prophylaxis in mice using embryonic stem cells and carbon nanotubes. Int J Nanomedicine. 2011;1945. doi:10.2147/IJN.S24060

60. Hassan HAFM, Smyth L, Rubio N, et al. Carbon nanotubes' surface chemistry determines their potency as vaccine nanocarriers in vitro and in vivo. $J$ Control Release. 2016;225:205-216. doi:10.1016/j.jconrel.2016.01.030

61. Mu Q, Broughton DL, Yan B. Endosomal Leakage and nuclear translocation of multiwalled carbon nanotubes: developing a model for cell uptake. Nano Lett. 2009;9(12):4370-4375. doi: $10.1021 / \mathrm{n} 1902647 \mathrm{x}$

62. Al-Jamal KT, Nerl H, Müller KH, et al. Cellular uptake mechanisms of functionalised multi-walled carbon nanotubes by $3 \mathrm{D}$ electron tomography imaging. Nanoscale. 2011;3(6):2627. doi: $10.1039 / \mathrm{c} 1 \mathrm{nr} 10080 \mathrm{~g}$

63. Behzadi S, Serpooshan V, Tao W, et al. Cellular uptake of nanoparticles: journey inside the cell. Chem Soc Rev. 2017;46 (14):4218-4244. doi:10.1039/C6CS00636A

64. Maruyama K, Haniu H, Saito N, et al. Endocytosis of Multiwalled carbon nanotubes in bronchial epithelial and mesothelial cells. Biomed Res Int. 2015;2015:1-9. doi:10.1155/2015/793186

65. Cui X, Wan B, Yang Y, Ren X, Guo L-H. Length effects on the dynamic process of cellular uptake and exocytosis of single-walled carbon nanotubes in murine macrophage cells. Sci Rep. 2017;7(1):1518. doi:10.1038/s41598-017-01746-9

66. Magrez A, Kasas S, Salicio V, et al. Cellular toxicity of carbon-based nanomaterials. Nano Lett. 2006;6(6):1121-1125. doi:10.1021/n1060162e

67. Hirano S, Fujitani Y, Furuyama A, Kanno S. Macrophage receptor with collagenous structure (MARCO) is a dynamic adhesive molecule that enhances uptake of carbon nanotubes by CHO-K1 Cells. Toxicol Appl Pharmacol. 2012;259(1):96-103. doi:10. 1016/j.taap.2011.12.012

68. Yang $M$, Zhang $M$, Nakajima $H$, Yudasaka $M$, Iijima $S$, Okazaki T. Time-dependent degradation of carbon nanotubes correlates with decreased reactive oxygen species generation in macrophages. Int J Nanomedicine. 2019;14:2797-2807. doi:10. 2147/IJN.S199187

69. Ma H, Zhang X, Yang Y, et al. Cellular uptake, organelle enrichment, and in vitro antioxidation of fullerene derivatives, mediated by surface charge. Langmuir. 2021;37(8):2740-2748. doi:10.10 21/acs.langmuir.0c03483

70. Zhu W, von Dem Bussche A, Yi X, et al. Nanomechanical mechanism for lipid bilayer damage induced by carbon nanotubes confined in intracellular vesicles. Proc Natl Acad Sci. 2016;113 (44):12374-12379. doi:10.1073/pnas.1605030113 
71. Kraszewski S, Bianco A, Tarek M, Ramseyer C. Insertion of short amino-functionalized single-walled carbon nanotubes into phospholipid bilayer occurs by passive diffusion. PLoS One. 2012;7 (7):e40703. doi:10.1371/journal.pone.0040703

72. Lin J, Miao L, Zhong G, Lin C-H, Dargazangy R, AlexanderKatz A. Understanding the synergistic effect of physicochemical properties of nanoparticles and their cellular entry pathways. Commun Biol. 2020;3(1):205. doi:10.1038/s42003-020-0917-1

73. Costa PM, Bourgognon M, Wang JT-W, Al-Jamal KT. Functionalised carbon nanotubes: from intracellular uptake and cell-related toxicity to systemic brain delivery. J Control Release. 2016;241:200-219. doi:10.1016/j.jconrel.2016.09.033

74. Bussy C, Cambedouzou J, Lanone S, et al. Carbon nanotubes in macrophages: imaging and chemical analysis by $\mathrm{x}$-ray fluorescence microscopy. Nano Lett. 2008;8(9):2659-2663. doi:10.1021/ nl800914m

75. Fadel TR, Fahmy TM. Immunotherapy applications of carbon nanotubes: from design to safe applications. Trends Biotechnol. 2014;32(4):198-209. doi:10.1016/j.tibtech.2014.02.005

76. Kraegeloh A, Suarez-Merino B, Sluijters T, Micheletti C. Implementation of safe-by-design for nanomaterial development and safe innovation: why we need a comprehensive approach. Nanomaterials. 2018;8(4):239. doi:10.3390/nano8040239

77. Allegri M, Perivoliotis DK, Bianchi MG, et al. Toxicity determinants of multi-walled carbon nanotubes: the relationship between functionalization and agglomeration. Toxicol Reports. 2016; 3:230-243. doi:10.1016/j.toxrep.2016.01.011

78. Zhou L, Forman HJ, Ge Y, Lunec J. Multi-walled carbon nanotubes: a cytotoxicity study in relation to functionalization, dose and dispersion. Toxicol Vitr. 2017;42:292-298. doi:10.1016/j. tiv.2017.04.027

79. Yehia HN, Draper RK, Mikoryak C, et al. Single-walled carbon nanotube interactions with HeLa cells. J Nanobiotechnology. 2007;5(1):8. doi:10.1186/1477-3155-5-8

80. Mehra NK, Jain K, Jain NK. Pharmaceutical and biomedical applications of surface engineered carbon nanotubes. Drug Discov Today. 2015;20(6):750-759. doi:10.1016/j.drudis.2015. 01.006

81. Cohignac V, Landry MJ, Ridoux A, et al. Carbon nanotubes, but not spherical nanoparticles, block autophagy by a shape-related targeting of lysosomes in murine macrophages. Autophagy. 2018;14(8):1323-1334. doi:10.1080/15548627.2018.1474993

82. Singh S, Vardharajula S, Tiwari P, et al. Functionalized carbon nanotubes: biomedical applications. Int $J$ Nanomedicine. 2012:5361. doi:10.2147/IJN.S35832

83. Mohammadi MR, Nojoomi A, Mozafari M, Dubnika A, Inayathullah M, Rajadas J. Nanomaterials engineering for drug delivery: a hybridization approach. J Mater Chem B. 2017;5 (22):3995-4018. doi:10.1039/C6TB03247H

84. Zhang Y, Wu L, Jiang C, Yan B. Reprogramming cellular signaling machinery using surface-modified carbon nanotubes. Chem Res Toxicol. 2015;28(3):296-305. doi:10.1021/tx500480d

85. Pescatori M, Bedognetti D, Venturelli E, et al. Functionalized carbon nanotubes as immunomodulator systems. Biomaterials. 2013;34(18):4395-4403. doi:10.1016/j.biomaterials.2013.02.052

86. Mukherjee SP, Bondarenko O, Kohonen P, et al. Macrophage sensing of single-walled carbon nanotubes via Toll-like receptors. Sci Rep. 2018;8(1):1115. doi:10.1038/s41598-01819521-9

87. Meunier E, Coste A, Olagnier D, et al. Double-walled carbon nanotubes trigger IL- $1 \beta$ release in human monocytes through Nlrp3 inflammasome activation. Nanomed Nanotechnol Biol Med. 2012;8(6):987-995. doi:10.1016/j.nano.2011.11.004

88. Delogu LG, Venturelli E, Manetti R, et al. Ex vivo impact of functionalized carbon nanotubes on human immune cells. Nanomedicine. 2012;7(2):231-243. doi:10.2217/nnm.11.101
89. Allen BL, Kichambare PD, Gou P, et al. Biodegradation of single-walled carbon nanotubes through enzymatic catalysis. Nano Lett. 2008;8(11):3899-3903. doi:10.1021/nl802315h

90. Kotchey GP, Zhao Y, Kagan VE, Star A. Peroxidase-mediated biodegradation of carbon nanotubes in vitro and in vivo. $A d v$ Drug Deliv Rev. 2013;65(15):1921-1932. doi:10.1016/j. addr.2013.07.007

91. Vlasova II, Kapralov AA, Michael ZP, et al. Enzymatic oxidative biodegradation of nanoparticles: mechanisms, significance and applications. Toxicol Appl Pharmacol. 2016;299:58-69. doi:10.10 16/j.taap.2016.01.002

92. Kotchey GP, Gaugler JA, Kapralov AA, Kagan VE, Star A. Effect of antioxidants on enzyme-catalysed biodegradation of carbon nanotubes. J Mater Chem B. 2013;1(3):302-309. doi:10.1039/ C2TB00047D

93. Vlasova II, Sokolov AV, Chekanov AV, Kostevich VA, Vasilyev VB. Myeloperoxidase-induced biodegradation of single-walled carbon nanotubes is mediated by hypochlorite. Russ J Bioorganic Chem. 2011;37(4):453-463. doi:10.1134/ S1068162011040157

94. Elgrabli D, Dachraoui W, Marmier H, et al. Intracellular degradation of functionalized carbon nanotube/iron oxide hybrids is modulated by iron via Nrf2 pathway. Sci Rep. 2017;7(1):40997. doi:10.1038/srep40997

95. Shvedova AA, Pietroiusti A, Fadeel B, Kagan VE. Mechanisms of carbon nanotube-induced toxicity: focus on oxidative stress. Toxicol Appl Pharmacol. 2012;261(2):121-133. doi:10.1016/j. taap.2012.03.023

96. Zhang M, Yang M, Morimoto T, et al. Size-dependent cell uptake of carbon nanotubes by macrophages: a comparative and quantitative study. Carbon N Y. 2018;127:93-101. doi:10.1016/j.carbon. 2017.10.085

97. Rankin LC, Artis D. Beyond host defense: emerging functions of the immune system in regulating complex tissue physiology. Cell. 2018;173(3):554-567. doi:10.1016/j.cell.2018.03.013

98. Villa CH, Dao T, Ahearn I, et al. Single-walled carbon nanotubes deliver peptide antigen into dendritic cells and enhance igg responses to tumor-associated antigens. ACS Nano. 2011;5 (7):5300-5311. doi:10.1021/nn200182x

99. Tkach AV, Shurin GV, Shurin MR, et al. Direct effects of carbon nanotubes on dendritic cells induce immune suppression upon pulmonary exposure. ACS Nano. 2011;5(7):5755-5762. doi:10. $1021 / \mathrm{nn} 2014479$

100. Aldinucci A, Turco A, Biagioli T, et al. Carbon nanotube scaffolds instruct human dendritic cells: modulating immune responses by contacts at the nanoscale. Nano Lett. 2013;13 (12):6098-6105. doi:10.1021/n1403396e

101. Sayes CM, Liang F, Hudson JL, et al. Functionalization density dependence of single-walled carbon nanotubes cytotoxicity in vitro. Toxicol Lett. 2006;161(2):135-142. doi:10.1016/j.toxlet.2005.08.011

102. Fadel TR, Look M, Staffier PA, Haller GL, Pfefferle LD, Fahmy TM. Clustering of stimuli on single-walled carbon nanotube bundles enhances cellular activation. Langmuir. 2010;26 (8):5645-5654. doi:10.1021/la902068z

103. Benincasa M, Pacor S, Wu W, Prato M, Bianco A, Gennaro R. Antifungal activity of amphotericin $\mathrm{B}$ conjugated to carbon nanotubes. ACS Nano. 2011;5(1):199-208. doi:10.1021/nn1023522

104. Murphy FA, Schinwald A, Poland CA, Donaldson K. The mechanism of pleural inflammation by long carbon nanotubes: interaction of long fibres with macrophages stimulates them to amplify pro-inflammatory responses in mesothelial cells. Part Fibre Toxicol. 2012;9(1):8. doi:10.1186/1743-8977-9-8

105. Hansen JS, Rosengren TS, Johansson HKL, et al. Preconceptional exposure to multiwalled carbon nanotubes suppresses antibody production in mouse offspring. Nanotoxicology. 2020;14(5):711-724. doi:10.1080/17435390.2020.1755468 
106. Scheinberg DA, McDevitt MR, Dao T, Mulvey JJ, Feinberg E, Alidori S. Carbon nanotubes as vaccine scaffolds. Adv Drug Deliv Rev. 2013;65(15):2016-2022. doi:10.1016/j.addr.2013.07.013

107. Versiani AF, Astigarraga RG, Rocha ESO, et al. Multi-walled carbon nanotubes functionalized with recombinant Dengue virus 3 envelope proteins induce significant and specific immune responses in mice. $J$ Nanobiotechnology. 2017;15(1):26. doi:10.1186/s12951-017-0259-4

108. Patra JK, Das G, Fraceto LF, et al. Nano based drug delivery systems: recent developments and future prospects. J Nanobiotechnology. 2018;16(1):71. doi:10.1186/s12951-0180392-8

109. Salvadormorales C, Flahaut E, Sim E, Sloan J, Hgreen M, Sim R. Complement activation and protein adsorption by carbon nanotubes. Mol Immunol. 2006;43(3):193-201. doi:10.1016/j. molimm.2005.02.006

110. Mellors J, Tipton T, Longet S, Carroll M. Viral Evasion of the complement system and its importance for vaccines and therapeutics. Front Immunol. 2020;11. doi:10.3389/fimmu. 2020.01450

111. Andersen AJ, Robinson JT, Dai H, Hunter AC, Andresen TL, Moghimi SM. Single-walled carbon nanotube surface control of complement recognition and activation. ACS Nano. 2013;7 (2):1108-1119. doi:10.1021/nn3055175

112. Hamad I, Christy hunter A, Rutt KJ, Liu Z, Dai H, Moein moghimi S. Complement activation by PEGylated single-walled carbon nanotubes is independent of $\mathrm{Clq}$ and alternative pathway turnover. Mol Immunol. 2008;45(14):3797-3803. doi:10.1016/j. molimm.2008.05.020

113. Pondman KM, Sobik M, Nayak A, et al. Complement activation by carbon nanotubes and its influence on the phagocytosis and cytokine response by macrophages. Nanomed Nanotechnol Biol Med. 2014;10(6):1287-1299. doi:10.1016/j.nano.2014.02.010

114. Nagaraju K, Reddy R, Reddy N. A review on protein functionalized carbon nanotubes. J Appl Biomater Funct Mater. 2015;13 (4):548. doi:10.5301/jabfm.5000231

115. Pondman KM, Salvador-Morales C, Paudyal B, Sim RB, Kishore U. Interactions of the innate immune system with carbon nanotubes. Nanoscale Horizons. 2017;2(4):174-186. doi:10.1039/ C6NH00227G

116. Moghimi SM, Hunter AC. Complement monitoring of carbon nanotubes. Nat Nanotechnol. 2010;5(6):382. doi:10.1038/ nnano.2010.109

117. Dong J, Ma Q. Type 2 immune mechanisms in carbon nanotube-induced lung fibrosis. Front Immunol. 2018;9. doi:10.3389/fimmu.2018.01120

118. Duke KS, Bonner JC. Mechanisms of carbon nanotube-induced pulmonary fibrosis: a physicochemical characteristic perspective. WIREs Nanomed Nanobiotechnol. 2018;10(3). doi:10.1002/ wnan. 1498

119. Mitchell LA, Lauer FT, Burchiel SW, McDonald JD. Mechanisms for how inhaled multiwalled carbon nanotubes suppress systemic immune function in mice. Nat Nanotechnol. 2009;4(7):451-456. doi:10.1038/nnano.2009.151

120. Hassan HAFM, Smyth L, Wang JT-W, et al. Dual stimulation of antigen presenting cells using carbon nanotube-based vaccine delivery system for cancer immunotherapy. Biomaterials. 2016;104:310-322. doi:10.1016/j.biomaterials.2016.07.005

121. Bianco A, Hoebeke J, Godefroy S, et al. Cationic Carbon Nanotubes Bind to $\mathrm{CpG}$ Oligodeoxynucleotides and Enhance Their Immunostimulatory Properties. J Am Chem Soc. 2005;127 (1):58-59. doi:10.1021/ja044293y

122. Li R, Wang X, Ji Z, et al. Surface charge and cellular processing of covalently functionalized multiwall carbon nanotubes determine pulmonary toxicity. ACS Nano. 2013;7(3):2352-2368. doi:10.1021/nn305567s
123. Reed SG, Orr MT, Fox CB. Key roles of adjuvants in modern vaccines. Nat Med. 2013;19(12):1597-1608. doi:10.1038/nm.3409

124. Baylor NW. The Regulatory Evaluation of Vaccines for Human Use. New York, NY: Humana Press; 2016:773-787.

125. Lambrecht BN, Kool M, Willart MA, Hammad H. Mechanism of action of clinically approved adjuvants. Curr Opin Immunol. 2009;21(1):23-29. doi:10.1016/j.coi.2009.01.004

126. Petrovsky N. Comparative safety of vaccine adjuvants: a summary of current evidence and future needs. Drug Saf. 2015;38(11):1059-1074. doi:10.1007/s40264-015-0350-4

127. O'Hagan DT, Lodaya RN, Lofano G. The continued advance of vaccine adjuvants - 'we can work it out.'. Semin Immunol. 2020;50:101426. doi:10.1016/j.smim.2020.101426

128. Del Giudice G, Rappuoli R, Didierlaurent AM. Correlates of adjuvanticity: a review on adjuvants in licensed vaccines. Semin Immunol. 2018;39:14-21. doi:10.1016/j.smim.2018.05.001

129. Kool M, Soullié T, van Nimwegen M, et al. Alum adjuvant boosts adaptive immunity by inducing uric acid and activating inflammatory dendritic cells. J Exp Med. 2008;205(4):869-882. doi:10.1084/jem.20071087

130. Díez-Domingo J, Baldó J-M, Planelles-Catarino MV, et al. Phase II, randomized, open, controlled study of AS03-adjuvanted H5N1 pre-pandemic influenza vaccine in children aged 3 to 9 years: follow-up of safety and immunogenicity persistence at 24 months post-vaccination. Influenza Other Respi Viruses. 2015;9(2):68-77. doi:10.1111/irv.12295

131. Ensoli B, Cafaro A, Monini P, Marcotullio S, Ensoli F. Challenges in HIV vaccine research for treatment and prevention. Front Immunol. 2014;5(SEP):1-11. doi:10.3389/fimmu.2014.00417

132. Parra J, Abad-Somovilla A, Mercader JV, Taton TA, Abad-Fuentes A. Carbon nanotube-protein carriers enhance size-dependent self-adjuvant antibody response to haptens. $J$ Control Release. 2013;170(2):242-251. doi:10.1016/j.jconrel.2013.05.019

133. Pantarotto D, Partidos CD, Hoebeke J, et al. Immunization with peptide-functionalized carbon nanotubes enhances virus-specific neutralizing antibody responses. Chem Biol. 2003;10 (10):961-966. doi:10.1016/j.chembiol.2003.09.011

134. Meng J, Meng J, Duan J, et al. Carbon Nanotubes conjugated to tumor lysate protein enhance the efficacy of an antitumor immunotherapy. Small. 2008;4(9):1364-1370. doi:10.1002/smll. 200701059

135. Zeinali M, Jammalan M, Ardestani SK, Mosaveri N. Immunological and cytotoxicological characterization of tuberculin purified protein derivative (PPD) conjugated to single-walled carbon nanotubes. Immunol Lett. 2009;126(1-2):48-53. doi:10. 1016/j.imlet.2009.07.012

136. Dao T, Yan S, Veomett N, et al. Targeting the Intracellular WT1 oncogene product with a therapeutic human antibody. Sci Transl Med. 2013;5(176):176ra33-176ra33. doi:10.1126/scitranslmed.30 05661

137. Thi Mai Hoa L. Characterization of multi-walled carbon nanotubes functionalized by a mixture of $\mathrm{HNO} 3 / \mathrm{H} 2 \mathrm{SO} 4$. Diam Relat Mater. 2018;89:43-51. doi:10.1016/j.diamond.2018.08.008

138. Vlasova II, Vakhrusheva TV, Sokolov AV, et al. PEGylated single-walled carbon nanotubes activate neutrophils to increase production of hypochlorous acid, the oxidant capable of degrading nanotubes. Toxicol Appl Pharmacol. 2012;264(1):131-142. doi:10.1016/j.taap.2012.07.027

139. Yuan X, Zhang X, Sun L, Wei Y, Wei X. Cellular Toxicity and immunological effects of carbon-based nanomaterials. Part Fibre Toxicol. 2019;16(1):18. doi:10.1186/s12989-019-0299-z

140. Chowdhry A, Kaur J, Khatri M, Puri V, Tuli R, Puri S. Characterization of functionalized multiwalled carbon nanotubes and comparison of their cellular toxicity between HEK 293 cells and zebra fish in vivo. Heliyon. 2019;5(10):e02605. doi:10.1016/ j.heliyon.2019.e02605 
141. Gregory AE, Williamson ED, Prior JL, et al. Conjugation of Y. pestis F1-antigen to gold nanoparticles improves immunogenicity. Vaccine. 2012;30(48):6777-6782. doi:10.1016/ j.vaccine.2012.09.021

142. Fadel TR, Steenblock ER, Stern E, et al. Enhanced cellular activation with single walled carbon nanotube bundles presenting antibody stimuli. Nano Lett. 2008;8(7):2070-2076. doi:10.1021/n1080332i

143. Gottardi R, Douradinha B. Carbon nanotubes as a novel tool for vaccination against infectious diseases and cancer. J Nanobiotechnology. 2013;11(1):30. doi:10.1186/1477-3155-11-30

144. Gratton SEA, Ropp PA, Pohlhaus PD, et al. The effect of particle design on cellular internalization pathways. Proc Natl Acad Sci. 2008;105(33):11613-11618. doi:10.1073/pnas.0801763105

145. Waibl F, Fernández-Quintero ML, Kamenik AS, et al. Conformational ensembles of antibodies determine their hydrophobicity. Biophys $J$. 2021;120(1):143-157. doi:10.1016/j.bpj.2020.11.010

146. Cheng HKF, Basu T, Sahoo NG, Li L, Chan SH. Current advances in the carbon nanotube/thermotropic main-chain liquid crystalline polymer nanocomposites and their blends. Polymers. 2012;4(2):889-912. doi:10.3390/polym4020889

147. Quang NH, Kim D-H. Control of growth mode of multiwalled carbon nanotubes. $J$ Phys Conf Ser. 2009;187:012065. doi:10.1088/1742-6596/187/1/012065

148. Das R, Shahnavaz Z, Ali ME, Islam MM, Abd Hamid SB. Can we optimize arc discharge and laser ablation for well-controlled carbon nanotube synthesis? Nanoscale Res Lett. 2016;11(1):510. doi:10.1186/s11671-016-1730-0

149. Szabó A, Perri C, Csató A, Giordano G, Vuono D, Nagy JB. Synthesis methods of carbon nanotubes and related materials. Materials. 2010;3(5):3092-3140. doi:10.3390/ma3053092

150. Saleh DM, Alexander WT, Numano T, et al. Comparative carcinogenicity study of a thick, straight-type and a thin, tangled-type multi-walled carbon nanotube administered by intra-tracheal instillation in the rat. Part Fibre Toxicol. 2020;17(1):48. doi:10.1186/s12989-020-00382-y

151. Wang X, Xia T, Addo Ntim S, et al. Dispersal state of multiwalled carbon nanotubes elicits profibrogenic cellular responses that correlate with fibrogenesis biomarkers and fibrosis in the murine lung. ACS Nano. 2011;5(12):9772-9787. doi:10.1021/nn2033055

152. Sakamoto Y, Hojo M, Kosugi Y, et al. Comparative study for carcinogenicity of 7 different multi-wall carbon nanotubes with different physicochemical characteristics by a single intraperitoneal injection in male Fischer 344 rats. $J$ Toxicol Sci. 2018;43 (10):587-600. doi:10.2131/jts.43.587
153. Eatemadi A, Daraee H, Karimkhanloo H, et al. Carbon nanotubes: properties, synthesis, purification, and medical applications. Nanoscale Res Lett. 2014;9(1):393. doi:10.1186/1556-276X-9-393

154. Borzooeian Z, Taslim ME, Ghasemi O, Rezvani S, Borzooeian G, Nourbakhsh A. A high precision method for length-based separation of carbon nanotubes using bio-conjugation, SDS-PAGE and silver staining. Ceña Ved. PLoS One. 2018;13(6):e0197972. doi:10.1371/journal.pone.0197972

155. Yang S-T, Luo J, Zhou Q, Wang H. Pharmacokinetics, metabolism and toxicity of carbon nanotubes for biomedical purposes. Theranostics. 2012;2(3):271-282. doi:10.7150/thno.3618

156. Bardhan NM. 30 years of advances in functionalization of carbon nanomaterials for biomedical applications: a practical review. J Mater Res. 2017;32(1):107-127. doi:10.1557/jmr.2016.449

157. Serpell CJ, Kostarelos K, Davis BG. Can carbon nanotubes deliver on their promise in biology? Harnessing unique properties for unparalleled applications. ACS Cent Sci. 2016;2(4):190-200. doi:10.1021/acscentsci.6b00005

158. Palomäki J, Välimäki E, Sund J, et al. Long, Needle-like Carbon Nanotubes and Asbestos Activate the NLRP3 Inflammasome through a Similar Mechanism. ACS Nano. 2011;5(9):6861-6870. doi:10.1021/nn200595c

159. Sharma CS, Sarkar S, Periyakaruppan A, et al. Single-walled carbon nanotubes induces oxidative stress in rat lung epithelial cells. J Nanosci Nanotechnol. 2007;7(7):2466-2472. doi:10.1166/ jnn.2007.431

160. Mohanta D, Patnaik S, Sood S, Das N. Carbon nanotubes: evaluation of toxicity at biointerfaces. J Pharm Anal. 2019;9 (5):293-300. doi:10.1016/j.jpha.2019.04.003

161. Khan HA, Khan I, Lee Y. Role of immune factors in bioavailability and toxicity of carbon nanomaterials. In: Fullerens, Graphenes and Nanotubes. Elsevier; 2018:601-630. doi:10. 1016/B978-0-12-813691-1.00015-4

162. Khang D, Lee S, Kim S-H. High dispersity of carbon nanotubes diminishes immunotoxicity in spleen. Int $J$ Nanomedicine. 2015;2697. doi:10.2147/IJN.S80836
International Journal of Nanomedicine

\section{Publish your work in this journal}

The International Journal of Nanomedicine is an international, peerreviewed journal focusing on the application of nanotechnology in diagnostics, therapeutics, and drug delivery systems throughout the biomedical field. This journal is indexed on PubMed Central, MedLine, CAS, SciSearch ${ }^{\mathbb{R}}$, Current Contents ${ }^{\mathbb{B}} /$ Clinical Medicine,
Journal Citation Reports/Science Edition, EMBase, Scopus and the Elsevier Bibliographic databases. The manuscript management system is completely online and includes a very quick and fair peer-review system, which is all easy to use. Visit http://www.dovepress.com/ testimonials.php to read real quotes from published authors. 